\title{
On precessing flow in an oblate spheroid of arbitrary eccentricity
}

\author{
By KEKE ZHANG ${ }^{1}$ KIT H. CHA N \\ AND XINHAO LIA O \\ ${ }^{1}$ Department of Mathematical Sciences, University of Exeter, EX4 4QF, UK \\ ${ }^{2}$ Department of Mathematics, University of Hong Kong, Pokfulam, Hong Kong \\ ${ }^{3}$ Key Laboratory of Planetary Sciences, Shanghai Astronomical Observatory, Chinese \\ Academy of Sciences, Shanghai 200030, China
}

(Received ?? and in revised form ??)

We consider a homogeneous fluid of viscosity $\nu$ confined within an oblate spheroidal cavity of arbitrary eccentricity $\mathcal{E}$ marked by the equatorial radius $d$ and the polar radius $d \sqrt{1-\mathcal{E}^{2}}$ with $0<\mathcal{E}<1$. The spheroidal container rotates rapidly with an angular velocity $\boldsymbol{\Omega}_{0}$ about its symmetry axis and precesses slowly with an angular velocity $\boldsymbol{\Omega}_{p}$ about an axis that is fixed in space. It is through both topographical and viscous effects that the spheroidal container and the viscous fluid are coupled together, driving precessing flow against viscous dissipation. The precessionally driven flow is characterized by three dimensionless parameters: the shape parameter $\mathcal{E}$, the Ekman number $E k=\nu /\left(d^{2}\left|\boldsymbol{\Omega}_{0}\right|\right)$ and the Poincaré number $P o= \pm\left|\boldsymbol{\Omega}_{p}\right| /\left|\boldsymbol{\Omega}_{0}\right|$. We derive a time-dependent asymptotic solution for the weakly precessing flow in the mantle frame of reference satisfying the non-slip boundary condition and valid for a spheroidal cavity of arbitrary eccentricity at $E k \ll 1$. No prior assumptions about the spatial-temporal structure of the precessing flow are made in the asymptotic analysis. We also carry out direct numerical simulation for both the weakly and the strongly precessing flow in the same frame of reference using a finite element method that is particularly suitable for non-spherical geometry. A satisfactory agreement between the asymptotic solution and direct numerical simulation is achieved for sufficiently small Ekman and Poincaré numbers. When the nonlinear effect is weak with $|P o| \ll 1$, the precessing flow in an oblate spheroid is characterized by an azimuthally travelling wave without having a mean azimuthal flow. Stronger nonlinear effects with increasing $|P o|$ produce a large-amplitude, time-independent mean azimuthal flow that is always westward in the mantle frame of reference. Implications of the precessionally driven flow for the westward motion observed in the Earth's fluid core are also discussed.

\section{Introduction}

Recent computations of the thermal and electrical conductivities of liquid iron mixtures at the Earth's core conditions suggest that both the conductivities are two to three times higher than previous estimates, which severely restricts the thermal power available for sustaining the geodynamo (Pozzo et al. 2012). This restriction reminds us of an alternative cause for generating and maintaining the geomagnetic field: the lunar-solar precession-driven flow in the Earth's liquid core of oblate spheroidal shape (see, for example, Bullard 1949; Malkus 1968). Kerswell (1996) estimated that there exists abundant precessional energy to drive the geodynamo while Tilgner (2005) and $\mathrm{Wu}$ and Roberts 
(2009) showed that the precession-driven flow can generate and sustain magnetic fields. In addition to the geodynamo, the lunar dynamo may be once driven by precession in its liquid core Dwyer at el. (2007). It is the abundant energy and the persistent nature of precession in an oblate spheroid that make it geophysically significant.

Over the past several decades, the problem of precessing flow in spheres or spheroids has been extensively studied. In experimental studies of the problem, the various structures of precession-driven flow are observed in weakly and strongly precessing systems (see, for example, Vanyo et al. 1995; Noir et al. 2001, 2003; Goto et al. 2007). Since the rotation axis of a fluid sphere in laboratory experiments is typically not parallel to the rotation axis of the Earth, the resulting weakly precessing flow is recently identified in spherical rotating laboratory experiments (Boisson et al. 2012; Triana et al. 2012). In theoretical studies of the problem, a frame of reference rotating about the precession axis is usually adopted and the precessing flow is assumed to be stationary and in the form of rigidbody rotation so as to simplify the mathematical analysis (Stewartson and Roberts 1963; Roberts and Stewartson 1965; Busse 1968). Furthermore, an oblate spheroid is usually assumed to have small eccentricity such that its departure from spherical geometry can be treated as a small perturbation in the analysis. A relatively simple torque-balance approach, by taking advantage of the rigid-body-rotation assumption, is also employed to study the precessing flow (Vanyo and Likins 1972; Noir et al. 2003), and Cébron et al. (2010) investigated the properties of the tilt-over mode in a precessing triaxial ellipsoid. In particular, Busse (1968) incorporated the weakly nonlinear effect within the viscous boundary layer and established the existence of a weak differential rotation in the precession frame. The asymptotic analysis of Zhang et al. (2010) concentrated on the time-dependent asymptotic solution in the mantle frame of reference for a precessing sphere, whilst Kida (2011) focused on the analysis of the conical shear layer in the critical latitudes of a precessing sphere in the precession frame. Instabilities breaking the antipodal symmetry of the primary flow in precessing spheres and spherical shells with small inner cores are recently examined by Hollerbach et al. (2013); see also relevant discussions by Lorenzani and Tilgner (2001). In numerical studies of the problem, spectral methods based on spherical harmonic expansions are usually adopted (see, for example, Hollerbach and Kerswell 1995; Tilgner and Busse 2001; Lorenzani and Tilgner 2001). An interesting problem about the interaction of stratification with precession in spherical geometry is recently examined by Wei and Tilgner (2013) using spectral methods.

The present study attempts to improve our understanding of the precession problem through (i) deriving an asymptotic solution for weakly precessing flow in an oblate spheroidal cavity of arbitrary eccentricity and (ii) simulating both weakly and strongly precessing flow using a finite element method that is particularly suitable for nonspherical geometry. In our asymptotic analysis, the mantle frame of reference together with oblate spheroidal coordinates will be adopted and, more significantly, no prior assumptions about the spatial-temporal structure of the flow will be made. It is noteworthy that an analytical study postulating that precessing flow is not only stationary but also in the form of rigid-body rotation in the precession frame is generally easier; see the relevant discussion by Tilgner (2007). Since nearly all the theoretical and numerical studies of rotating convection and convection-driven dynamos are conducted in the mantle frame of reference and since most geophysical observations are made in the Earth's mantle frame, it is desirable to adopt the mantle frame of reference in the theoretical analysis of precessing flow. In this connection, it should be noted that there exists no simple transformation between a stationary solution in the precession frame and a time-dependent solution in the mantle frame.

The precessing spheroidal container and the viscous fluid are coupled via both topo- 


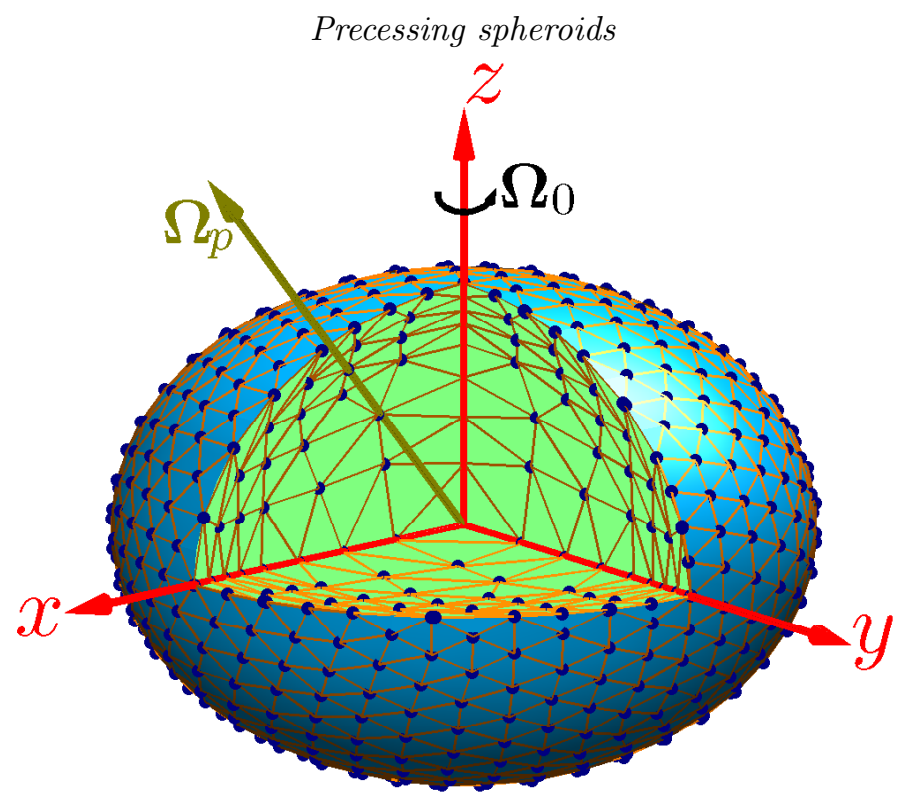

FIGURE 1. Geometry of a precessing oblate spheroid of arbitrary eccentricity $\mathcal{E}$ with equatorial radius $d$ and polar radius $d \sqrt{1-\mathcal{E}^{2}}$. The spheroid rotates rapidly with an angular velocity $\boldsymbol{\Omega}_{0}=\Omega_{0} \hat{\mathbf{z}}$ around the symmetry axis $z$ and precesses slowly with an angular velocity $\boldsymbol{\Omega}_{p}$ that is fixed in space with $\left|\boldsymbol{\Omega}_{p}\right| /\left|\boldsymbol{\Omega}_{0}\right| \ll 1$. The angle between $\boldsymbol{\Omega}_{0}$ and $\boldsymbol{\Omega}_{p}$ is denoted by $\alpha_{p}$ $\left(0<\alpha_{p} \leqslant \pi / 2\right)$. A schematic of the spheroidal finite element meshes used for direct numerical simulation is also illustrated, showing a denser mesh in the vicinity of the spheroidal bounding surface.

graphical and viscous effects, which drive a precessing flow against viscous dissipation. There are three dimensionless parameters - the shape parameter $\mathcal{E}$ (eccentricity) of the container, the Ekman number $E k$ and the Poincaré number $P o$ - that characterize the precessing flow in an oblate spheroidal cavity. In this study, we shall first derive a timedependent asymptotic solution of the weakly precessing flow in the mantle frame of reference valid only for $0<E k \ll 1$. We shall then carry out direct numerical simulation for both weakly and strongly precessing flow in the same frame of reference. By comparing the asymptotic solution to the result of numerical simulation, we demonstrate that a satisfactory agreement between the asymptotic solution and the numerical simulation is achieved for sufficiently small Ekman and Poincaré numbers $(0<|P o| \ll 1$ and $0<E k \ll 1)$ in an oblate spheroidal cavity of arbitrary eccentricity $(0<\mathcal{E}<1)$. At the same time, we shall reveal some interesting properties of the strongly nonlinear precessing flow in an oblate spheroidal cavity of arbitrary eccentricity.

In what follows we begin by presenting the governing mathematical equations of the problem in $\S 2$. The asymptotic analysis for weakly precessing flow in an oblate spheroidal cavity of arbitrary eccentricity is discussed in $\$ 3$ while the results of direct numerical simulation for both weakly and strongly precessing flow are presented in $\S 4$. A summary and some remarks highlighting a possible connection between the Earth's precession and the observed westward motion in the Earth's fluid core are given in $\S 5$. 


\section{Mathematical formulation of the problem}

Consider a viscous, incompressible and homogeneous fluid of density $\rho$ occupying an oblate spheroidal cavity of arbitrary eccentricity $\mathcal{E}$ described by the equation

$$
\frac{x^{2}}{d^{2}}+\frac{y^{2}}{d^{2}}+\frac{z^{2}}{d^{2}\left(1-\mathcal{E}^{2}\right)}=1
$$

where $0 \leqslant \mathcal{E}<1$ and the $z$-axis represents the symmetry axis of the oblate spheroid, which is illustrated in Fig. 1. Cartesian coordinates $(x, y, z)$, with the corresponding unit vectors $(\hat{\mathbf{x}}, \hat{\mathbf{y}}, \hat{\mathbf{z}})$, are fixed in the spheroidal container. In order to develop the theory of viscous boundary layers located on the bounding surface of a spheroidal cavity, it is mathematically convenient to introduce oblate spheroidal coordinates $(\eta, \phi, \tau)$, with the corresponding unit vectors $(\hat{\boldsymbol{\eta}}, \hat{\boldsymbol{\phi}}, \hat{\boldsymbol{\tau}})$, which are related to rectangular Cartesian coordinates $(x, y, z)$ by

$$
\begin{aligned}
& x^{2}=\left(\mathcal{E}^{2}+\eta^{2}\right)\left(1-\tau^{2}\right) \cos ^{2} \phi, \\
& y^{2}=\left(\mathcal{E}^{2}+\eta^{2}\right)\left(1-\tau^{2}\right) \sin ^{2} \phi, \\
& z^{2}=\eta^{2} \tau^{2} .
\end{aligned}
$$

In oblate spheroidal coordinates, the surfaces of constant $\eta$ form oblate spheroids providing a set of coordinate surfaces with the foci of all the oblate spheroids being located at $\sqrt{x^{2}+y^{2}}=\mathcal{E}$ and $z=0$ while the surfaces of constant $\tau$ form hyperboloids offering another set of coordinate surfaces with the foci of all the hyperboloids being also located at $\sqrt{x^{2}+y^{2}}=\mathcal{E}$ and $z=0$. The envelope $\mathcal{S}$ of the spheroidal cavity is simply given by $\eta=\sqrt{1-\mathcal{E}^{2}}$. Three different coordinates will be employed: Cartesian coordinates $(x, y, z)$ for direct numerical simulation, cylindrical coordinates $(s, \phi, z)$ and spheroidal coordinates $(\eta, \phi, \tau)$ for deriving an asymptotic solution of the spheroidal precessing flow. Note that the value of $\mathcal{E}$ is fixed by the envelope of a spheroidal cavity and that the limit $\mathcal{E} \rightarrow 0$ represents the special case of spherical geometry.

Suppose that the spheroidal container, depicted in Fig. 1, rotates rapidly with an angular velocity $\hat{\mathbf{z}} \Omega_{0}$ and, at the same time, precesses slowly with an angular velocity $\boldsymbol{\Omega}_{p}$ that is fixed in space and inclined at an angle $\alpha_{p}\left(0<\alpha_{p} \leqslant \pi / 2\right)$ to $\hat{\mathbf{z}}$. In comparison to spherical geometry, fluid motion in the precessing spheroid of arbitrary eccentricity $0<\mathcal{E}<1$ is driven through both viscous and topographic coupling between the container and the viscous fluid. It is noteworthy that we choose the geometry of an oblate spheroid because many planets and stars are rotating rapidly and, consequently, their shape, distorted by the strong effect of rotation, is described by a oblate spheroid (see, for example, Kong et al. 2010, 2013). On scaling the governing equations with the major semi-axis $d$ as the length scale, $\Omega_{0}^{-1}$ as the unit of time and $\rho d^{2} \Omega_{0}^{2}$ as the unit of pressure, we obtain the dimensionless equations in the mantle frame of reference

$$
\begin{aligned}
\frac{\partial \mathbf{u}}{\partial t}+\mathbf{u} \cdot \nabla \mathbf{u}+2\left(\hat{\mathbf{z}}+P o \widehat{\boldsymbol{\Omega}}_{p}\right) \times \mathbf{u} & =-\nabla P+E k \nabla^{2} \mathbf{u}+P o \mathbf{r} \times\left(\widehat{\boldsymbol{\Omega}}_{p} \times \hat{\mathbf{z}}\right) \\
\nabla \cdot \mathbf{u} & =0
\end{aligned}
$$

where $P$ is the reduced pressure containing all the gradient terms and $\widehat{\boldsymbol{\Omega}}_{p}$ denotes the non-dimensional, time-dependent precession vector given by

$$
\widehat{\boldsymbol{\Omega}}_{p}=\sin \alpha_{p}(\hat{\mathbf{x}} \cos t-\hat{\mathbf{y}} \sin t)+\hat{\mathbf{z}} \cos \alpha_{p},
$$

which gives rise to

$$
\mathbf{r} \times\left(\widehat{\boldsymbol{\Omega}}_{p} \times \hat{\mathbf{z}}\right)=-\nabla\left[z s \sin \alpha_{p} \cos (\phi+t)\right]-\hat{\mathbf{z}}\left[2 s \sin \alpha_{p} \cos (\phi+t)\right] .
$$


In equation (2.1), $\mathbf{u}$ is the three-dimensional velocity field, the Ekman number, $E k=$ $\nu / \Omega_{0} d^{2}$, provides the measure of relative importance between the typical viscous force and the Coriolis force, and the Poincaré number, $P o= \pm\left|\Omega_{p}\right| / \Omega_{0}$, quantifies the strength of the precessional forcing. Positive $P o$ corresponds to the case of prograde precession while $P o<0$ for retrograde precession. The precessing flow on the bounding surface, $\mathcal{S}$, of the spheroidal container in the mantle frame of reference is at rest, which requires

$$
\hat{\mathbf{n}} \cdot \mathbf{u}=\hat{\mathbf{n}} \times \mathbf{u}=0,
$$

where $\hat{\mathbf{n}}$ denotes the outward unit normal to the bounding surface $\mathcal{S}$ of the spheroid. The last term on the right-hand side of (2.1) is known as the Poincaré force which drives precessional flow against viscous dissipation.

When $|P o|$ is sufficiently small $(0<|P o| \ll 1)$ such that the weakly precessing flow has a small amplitude $|\mathbf{u}|=\mathrm{O}(\epsilon) \ll 1$, (2.1) can be linearised for an arbitrary precession angle $\alpha_{p}$ by omitting the quadratic and product terms, which are $\mathbf{u} \cdot \nabla \mathbf{u}=\mathrm{O}\left(\epsilon^{2}\right)$ and small perturbations to the Coriolis force

$$
\left|P o \widehat{\boldsymbol{\Omega}}_{p} \times \mathbf{u}\right|=\mathrm{O}\left(\left|P_{o}\right| \epsilon\right) .
$$

The nonlinear equations (2.1)-(2.2) can be then linearized to yield

$$
\begin{aligned}
\frac{\partial \mathbf{u}}{\partial t}+2 \hat{\mathbf{z}} \times \mathbf{u} & =-\nabla p+E k \nabla^{2} \mathbf{u}-\hat{\mathbf{z}}\left(2 s P o \sin \alpha_{p} \mathrm{e}^{\mathrm{i} \phi}\right) \mathrm{e}^{\mathrm{i} t}, \\
\nabla \cdot \mathbf{u} & =0,
\end{aligned}
$$

where $\mathrm{i}=\sqrt{-1}, p$ is a different reduced pressure and the real part of $\mathbf{u}$ will be taken as a physical solution. It should be noted that, by neglecting the term $P o \widehat{\boldsymbol{\Omega}}_{p} \times \mathbf{u}$ with the weak precession limit, the possible resonance discussed by Noir et al. (2003) and Busse (1968) is removed. However, this resonance term can be readily included; see Section 5 for the detailed discussion.

The linear problem - defined by (2.5)-(2.6) subject to the boundary conditions (2.4) - will be solved analytically in an oblate spheroidal cavity of arbitrary eccentricity with $0<\mathcal{E}<1$ for small Ekman numbers $0<E k \ll 1$. After deriving an asymptotic solution for the weakly precessing flow, we shall obtain solutions to the fully nonlinear equations (2.1)-(2.2) subject to the boundary conditions (2.4) through nonlinear direct numerical simulation using a finite element method.

\section{Asymptotic analysis in the mantle frame}

\subsection{Asymptotic expansion}

In our asymptotic analysis, the mantle frame of reference will be adopted and no prior assumptions about the spatial-temporal structure of the precessing flow will be made. In all that follows, $\mathbf{u}$ and $p$ are understood to mean the flow velocity and pressure seen in the mantle frame of reference.

The asymptotic analysis is based on the following physical and mathematical observations. First, all the explicitly analytical solutions to (2.5)-(2.6) in the limits $P o \rightarrow 0$ and $E k \rightarrow 0$, which describe unforced inertial wave modes in an oblate spheroid of arbitrary eccentricity with $0<\mathcal{E}<1$, are available (Zhang et al. 2004). An important unanswered mathematical question in the theory of rotating fluids has been the completeness of inertial waves or inertial modes(Greenspan 1968). Recently, Cui et al. (2013) is able to provide the first mathematical proof for the completeness of inertial wave modes in a 
rotating annular channel by establishing the completeness relation, or Parseval's equality, for any piecewise continuous, differentiable velocity $\mathbf{u}$ of an incompressible fluid. It is reasonable to postulate, even though a rigorous mathematical proof for spherical or spheroidal geometry does not exist in literature, that the set of the spheroidal inertial modes is also mathematically complete. This offers the necessary framework for the construction of a leading-order solution for time-dependent interior precessing flow without making any prior assumptions, which has been successfully applied to constructing the general asymptotic solution in a precessing circular cylinder (Liao and Zhang 2012a). In other words, the velocity $\mathbf{u}_{0}$ and the pressure $p_{0}$ for $0<|P o| \ll 1$ at $E k=0$, because the Poincaré forcing Por $\times\left(\widehat{\boldsymbol{\Omega}}_{p} \times \hat{\mathbf{z}}\right)$ in the mantle frame is diurnal, can always be written in the form

$$
\begin{aligned}
& \mathbf{u}_{0}(\eta, \phi, \tau, t)=\left[\sum_{m} \sum_{n} \sum_{k} \mathcal{A}_{m n k} \mathbf{u}_{m n k}(\eta, \phi, \tau)\right] \mathrm{e}^{\mathrm{i} t}, \\
& p_{0}(\eta, \phi, \tau, t)=\left[\sum_{m} \sum_{n} \sum_{k} \mathcal{A}_{m n k} p_{m n k}(\eta, \phi, \tau)\right] \mathrm{e}^{\mathrm{i} t},
\end{aligned}
$$

where $\mathcal{A}_{m n k}$ are complex coefficients to be determined, and the triple index notation - $m$ is the azimuthal wavenumber and $n$ and $k$ represent roughly the axial and radial wavenumbers respectively - is used, and $\mathbf{u}_{m n k}$ and $p_{m n k}$ are complex and represent the spatial part of an inertial mode in oblate spheroids satisfying

$$
2 \mathrm{i} \sigma_{m n k} \mathbf{u}_{m n k}(\eta, \phi, \tau)+2 \hat{\mathbf{z}} \times \mathbf{u}_{m n k}(\eta, \phi, \tau)+\nabla p_{m n k}(\eta, \phi, \tau)=0
$$

and

$$
\nabla \cdot \mathbf{u}_{m n k}(\eta, \phi, \tau)=0
$$

with the boundary condition

$$
\hat{\mathbf{n}} \cdot \mathbf{u}_{m n k}=0
$$

on the bounding surface $\mathcal{S}$ of the spheroid. Here $\sigma_{m n k}$ denotes the half-frequency of a spheroidal inertial mode $\mathbf{u}_{m n k}$ with $\left|\sigma_{m n k}\right|<1$.

Second, an asymptotic solution for $0<|P o| \ll 1$ and $0<E k \ll 1$ can be regarded as adding a small perturbation to $\left(\mathbf{u}_{0}, p_{0}\right)$ along with a thin viscous boundary layer, forming a mathematically tractable asymptotic problem in the mantle frame of reference. It follows that the velocity $\mathbf{u}$ and the pressure $p$ for the weakly precessing flow marked by $0<|P o| \ll 1$ and $0<E k \ll 1$ can always be expressible as

$$
\begin{aligned}
& \mathbf{u}=\left\{\left[\sum_{m n k} \mathcal{A}_{m n k} \mathbf{u}_{m n k}(\eta, \phi, \tau)\right]+\widehat{\mathbf{u}}(\eta, \tau, \phi)+\widetilde{\mathbf{u}}(\eta, \tau, \phi)\right\} \mathrm{e}^{\mathrm{i} t}, \\
& p=\left\{\left[\sum_{m n k} \mathcal{A}_{m n k} p_{m n k}(\eta, \phi, \tau)\right]+\widehat{p}(\eta, \tau, \phi)+\widetilde{p}(\eta, \tau, \phi)\right\} \mathrm{e}^{\mathrm{i} t},
\end{aligned}
$$

where viscous action on $\left(\mathbf{u}_{0}, p_{0}\right)$ induces a thin viscous boundary layer on $\mathcal{S}$, denoted by $\widetilde{\mathbf{u}}$ and $\widetilde{p}$. Furthermore, by producing a normal mass flux from, or sucking the interior fluid into, the thin viscous boundary layer, the viscous effect drives the secondary interior flow $\widehat{\mathbf{u}}$ and communicates to the interior fluid. Note that, apart from the mathematical completeness of the spheroidal inertial modes $\mathbf{u}_{m n k}$, no prior assumptions about the spatial structure of a precessing flow is made in the asymptotic expansions (3.1) and (3.2) for an oblate spheroid of arbitrary eccentricity. 


\subsection{The solvability condition}

Substituting (3.1) and (3.2) into (2.5), multiplying the resulting equation by $\mathbf{u}_{m n k}^{*}$, the complex conjugate of $\mathbf{u}_{m n k}$, and then integrating over the spheroid, we obtain the solvability condition of the problem:

$$
\begin{gathered}
\mathrm{i}\left(1-2 \sigma_{m n k}\right) \mathcal{A}_{m n k} \int_{\mathcal{V}}\left|\mathbf{u}_{m n k}\right|^{2} \mathrm{~d} \mathcal{V}+\int_{\mathcal{S}}\left\{\left[p_{m n k}^{*}\right]_{\mathcal{S}} \hat{\mathbf{n}} \cdot \widehat{\mathbf{u}}\right\} \mathrm{d} \mathcal{S} \\
=2 P o \sin \alpha_{p} \int_{\mathcal{V}}\left(s \mathbf{u}_{m n k}^{*} \cdot \hat{\mathbf{z}} \mathrm{e}^{\mathrm{i} \phi}\right) \mathrm{d} \mathcal{V},
\end{gathered}
$$

where $\int_{\mathcal{V}} \mathrm{d} \mathcal{V}$ denotes the volume integral over the spheroid, $\int_{\mathcal{S}} \mathrm{d} \mathcal{S}$ represents the surface integral over the bounding surface $\mathcal{S}$ of the spheroid, the flux $\hat{\mathbf{n}} \cdot \widehat{\mathbf{u}}$ in the surface integral is connected with the boundary flow $\widetilde{\mathbf{u}}, p_{m n k}^{*}$ denotes the complex conjugate of $p_{m n k}$ and $[f]_{\mathcal{S}}$ denotes the evaluation of $f$ at the bounding surface $\mathcal{S}$ and the indices, $m, n$ and $k$, take all permissible values.

A major task of our analysis is to derive an expression for all complex coefficients $\mathcal{A}_{\text {mnk }}$ by carrying out the three integrals in the solvability condition (3.3). While the first integral on the left-hand side of (3.3) is straightforward, the volume integral on the righthand side of (3.3) associated with the Poincaré forcing needs careful consideration. Since the Poincaré force, $\hat{\mathbf{z}}\left(2 s P_{o} \sin \alpha_{p}\right) \mathrm{e}^{\mathrm{i} \phi}$ in (2.5), is equatorially antisymmetric with respect to the plane at $z=0$ and azimuthally described by the azimuthal wavenumber $m=1$, only a subset of the spheroidal inertial modes that have the azimuthal wavenumber $m=1$ with the equatorial antisymmetry can be excited by the Poincaré forcing, i.e., $\mathcal{A}_{m n k}=$ 0 when $m \neq 1$. A spheroidal inertial mode is said to be equatorially antisymmetric if its pressure $p_{m n k}$ obeys the parity $p_{m n k}(s, z, \phi)=-p_{m n k}(s,-z, \phi)$. The subset of equatorially antisymmetric modes with $m=1$ required in evaluating the solvability condition (3.3) is given by

$$
\begin{aligned}
& p_{1 n k}=\sum_{i=0}^{k} \sum_{j=0}^{k-i} \mathcal{C}_{1 k i j} \sigma_{1 n k}^{2 i}\left(1-\sigma_{1 n k}^{2}\right)^{j} s^{2 j+1} z^{2 i+1} \mathrm{e}^{\mathrm{i} \phi}, \\
& \hat{\mathbf{s}} \cdot \mathbf{u}_{1 n k}=-\frac{\mathrm{i}}{2} \sum_{i=0}^{k} \sum_{j=0}^{k-i} \mathcal{C}_{1 k i j} \sigma_{1 n k}^{2 i}\left(1-\sigma_{1 n k}^{2}\right)^{j-1}\left(2 j \sigma_{1 n k}+1+\sigma_{1 n k}\right) s^{2 j} z^{2 i+1} \mathrm{e}^{\mathrm{i} \phi}, \\
& \hat{\mathbf{z}} \cdot \mathbf{u}_{1 n k}=\frac{\mathrm{i}}{2} \sum_{i=0}^{k} \sum_{j=0}^{k-i} \mathcal{C}_{1 k i j} \sigma_{1 n k}^{2 i-1}\left(1-\sigma_{1 n k}^{2}\right)^{j}(2 i+1) s^{2 j+1} z^{2 i} \mathrm{e}^{\mathrm{i} \phi}, \\
& \hat{\boldsymbol{\phi}} \cdot \mathbf{u}_{1 n k}=\frac{1}{2} \sum_{i=0}^{k} \sum_{j=0}^{k-i} \mathcal{C}_{1 k i j} \sigma_{1 n k}^{2 i}\left(1-\sigma_{1 n k}^{2}\right)^{j-1}\left(2 j+1+\sigma_{1 n k}\right) s^{2 j} z^{2 i+1} \mathrm{e}^{\mathrm{i} \phi},
\end{aligned}
$$

where $k \geqslant 0, n$ is restricted by $1 \leqslant n \leqslant(2 k+1)$ and

$$
C_{1 k i j}=\left[\frac{-1}{\left(1-\sigma_{1 n k}^{2} \mathcal{E}^{2}\right)}\right]^{i+j} \frac{[2(k+i+j)+3] ! !}{2^{j+1}(2 i+1) ! !(k-i-j) ! i ! j !(1+j) !} .
$$

The half frequencies of the spheroidal inertial modes in (3.4)-(3.7), $\sigma_{1 n k}$, are solutions of

$$
\begin{aligned}
0= & \sum_{j=0}^{k}(-1)^{j} \frac{[2(2 k+2-j)] !}{[2(k-j)+1] ! j !(2 k+2-j) !} \\
& \times\left[1-\frac{\left(1-\sigma_{1 n k}\right)[2(k-j)+1]}{\sigma_{1 n k}\left(1-\mathcal{E}^{2}\right)}\right]\left[\frac{\left(1-\mathcal{E}^{2}\right) \sigma_{1 n k}^{2}}{\left(1-\sigma_{1 n k}^{2} \mathcal{E}^{2}\right)}\right]^{k-j} .
\end{aligned}
$$


For any given $\mathcal{E}$ and $k$, there exist $(2 k+1)$ different real solutions for (3.8) corresponding to the $(2 k+1)$ different inertial modes (Zhang et al. 2004), which can be arranged in ascending order,

$$
0<\left|\sigma_{11 k}\right|<\left|\sigma_{12 k}\right|<\left|\sigma_{13 k}\right|, \ldots,<\left|\sigma_{1 n k}\right|<\ldots,
$$

where the index $n$ for $\sigma_{1 n k}$ denotes the $n$-th smallest root of (3.8) for given $k$.

Upon using the explicit expressions (3.5)-(3.7), the volume integration on the righthand side of (3.3) can be readily performed to yield

$$
2 P o \sin \alpha_{p} \int_{\mathcal{V}}\left(\mathbf{u}_{1 n k}^{*} \cdot \hat{\mathbf{z}} s \mathrm{e}^{\mathrm{i} \phi}\right) \mathrm{d} \mathcal{V}=4 \pi P o\left(1-\mathcal{E}^{2}\right)^{1 / 2} \sin \alpha_{p} \mathcal{I}_{n k},
$$

where $\mathcal{I}_{n k}$ denotes a two-dimensional summation for given $\sigma_{1 n k}$ and $\mathcal{E}$,

$$
\begin{aligned}
\mathcal{I}_{n k}=\sum_{i=0}^{k} \sum_{j=0}^{k-i} & (-1)^{i+j}\left[\frac{\sigma_{1 n k}^{2}\left(1-\mathcal{E}^{2}\right)}{\left(1-\sigma_{1 n k}^{2} \mathcal{E}^{2}\right)}\right]^{i} \\
& \times\left[\frac{\left(1-\sigma_{1 n k}^{2}\right)}{\left(1-\sigma_{1 n k}^{2} \mathcal{E}^{2}\right)}\right]^{j}\left[\frac{[2(k+i+j)+3] ! !}{(2 i+2 j+5) ! !(k-i-j) ! i ! j !}\right],
\end{aligned}
$$

with $k \geqslant 0$ and $1 \leqslant n \leqslant(2 k+1)$. In order to determine coefficients $\mathcal{A}_{1 n k}$ for all possible $n$ and $k$, we have to evaluate the summation $\mathcal{I}_{n k}$ for all permissible $n$ and $k$. When $k=0$ and $n=1$, a direct summation of $(3.9)$ together with $\sigma_{110}=1 /\left(2-\mathcal{E}^{2}\right)$ gives rise to

$$
\mathcal{I}_{10}=\frac{1}{5}
$$

When $k=1$, a direct summation of (3.9) can be also easily carried out, which yields

$$
\mathcal{I}_{n 1}=\sum_{i=0}^{1} \sum_{j=0}^{1-i}(-1)^{i+j}\left[\frac{\sigma_{1 n 1}^{2 i}\left(1-\sigma_{1 n 1}^{2}\right)^{j}}{\left(1-\sigma_{1 n 1}^{2} \mathcal{E}^{2}\right)^{i+j}}\right]\left[\frac{[2(k+i+j)+3] ! !\left(1-\mathcal{E}^{2}\right)^{i}}{(2 i+2 j+5) ! !(k-i-j) ! i ! j !}\right]=0
$$

for all permissible $\sigma_{1 n 1}$. When $k \geqslant 2$, the two indices $(i, j)$ in the summation (3.9) are so intimately entangled that direct summation becomes unmanageable. It can be, however, proven that $\mathcal{I}_{n k} \equiv 0$ for all $k \geqslant 2$. A key step in the proof is to establish a recurrence relationship that links the large $k$ summations of (3.9) with the small $k$ ones, like for $k=1$, that can be then evaluated directly. For this purpose, we introduce one additional index, $M$, by considering a new summation

$$
\begin{aligned}
\mathcal{P}_{n k}^{M} & =\sum_{i=0}^{k-M} \sum_{j=0}^{k-i-M}(-1)^{i+j}\left[\frac{\sigma_{1 n k}^{2}\left(1-\mathcal{E}^{2}\right)}{1-\sigma_{1 n k}^{2} \mathcal{E}^{2}}\right]^{i}\left(\frac{1-\sigma_{1 n k}^{2}}{1-\sigma_{1 n k}^{2} \mathcal{E}^{2}}\right)^{j} \\
& \times\left[\frac{[2(k+i+j)+3] ! !}{[2(i+j+M)+5] ! !(k-i-j-M) ! i ! j !}\right],
\end{aligned}
$$

where $k \geqslant 2$ with $(k-M) \geqslant 1$. Obviously,

$$
\mathcal{I}_{n k}=\mathcal{P}_{n k}^{0} \quad \text { at } \quad M=0
$$

Let

Then

$$
X_{n k}=\frac{\sigma_{1 n k}^{2}\left(1-\mathcal{E}^{2}\right)}{1-\sigma_{1 n k}^{2} \mathcal{E}^{2}}, 1-X_{n k}=\frac{1-\sigma_{1 n k}^{2}}{1-\sigma_{1 n k}^{2} \mathcal{E}^{2}}
$$

$$
\mathcal{P}_{n k}^{M}=\sum_{i=0}^{k-M} \sum_{j=0}^{k-i-M} \frac{(-1)^{i+j} X_{n k}^{i}\left(1-X_{n k}\right)^{j}[2(k+i+j)+3] ! !}{[2(i+j+M)+5] ! !(k-i-j-M) ! i ! j !} .
$$


For any $M \geqslant 0$ and $k \geqslant 2$ with $0 \leqslant M \leqslant(k-1),(3.11)$ can be rewritten in the form

$$
\begin{aligned}
\mathcal{P}_{n k}^{M} & =\left[\frac{2(M+1-k)}{(k-M)}\right] \\
& \times \sum_{i=0}^{k-(M+1)} \sum_{j=0}^{k-i-(M+1)} \frac{(-1)^{i+j} X_{n k}^{i}\left(1-X_{n k}\right)^{j}[2(k+i+j)+3] ! !}{[2(i+j+M+1)+5] ! ![k-i-j-(M+1)] ! i ! j !},
\end{aligned}
$$

suggesting that there exists a recurrence relationship between $\mathcal{P}_{n k}^{M}$ and $\mathcal{P}_{n k}^{M+1}$

$$
\mathcal{P}_{n k}^{M}=\left[\frac{2(M+1-k)}{k-M}\right] \mathcal{P}_{n k}^{M+1} .
$$

This means that

$$
\mathcal{P}_{n k}^{0}=\left[\frac{-2(k-1)}{k}\right] \mathcal{P}_{n k}^{1}=\cdots=\left[\frac{(-2)^{k-1}(k-1) !}{k !}\right] \mathcal{P}_{n k}^{k-1}
$$

or

$$
\mathcal{I}_{n k}=\mathcal{P}_{n k}^{0}=\left[\frac{(-2)^{k-1}}{k}\right] \mathcal{P}_{n k}^{k-1} .
$$

At $M=k-1$, the summation (3.10) can be easily carried out:

$$
\begin{aligned}
\mathcal{P}_{n k}^{k-1} & =\sum_{i=0}^{1} \sum_{j=0}^{1-i}(-1)^{i+j}\left[\frac{\sigma_{1 n k}^{2}\left(1-\mathcal{E}^{2}\right)}{1-\sigma_{1 n k}^{2} \mathcal{E}^{2}}\right]^{i}\left(\frac{1-\sigma_{1 n k}^{2}}{1-\sigma_{1 n k}^{2} \mathcal{E}^{2}}\right)^{j} \\
& \times\left[\frac{1}{(1-i-j) ! i ! j !}\right] \equiv 0 .
\end{aligned}
$$

By virtue of the recurrence relationship (3.12) and the summation (3.10) at $M=k-1$, we conclude that

$$
\mathcal{I}_{n k} \equiv 0 \text { for } k \geqslant 1,
$$

implying that coefficients $\mathcal{A}_{1 n k}$ are

$$
\mathcal{A}_{110} \neq 0 \text { but } \mathcal{A}_{1 n k}=0 \text { when } k \geqslant 1,1 \leqslant n \leqslant(2 k+1) .
$$

The solvability condition (3.3) then reduces to

$$
\begin{array}{r}
-\left(\frac{\mathrm{i} \mathcal{E}^{2}}{2-\mathcal{E}^{2}}\right) \mathcal{A}_{110} \int_{\mathcal{V}}\left|\mathbf{u}_{110}\right|^{2} \mathrm{~d} \mathcal{V}+\int_{\mathcal{S}}\left\{\left[p_{110}^{*}\right]_{\mathcal{S}} \hat{\mathbf{n}} \cdot \widehat{\mathbf{u}}\right\} \mathrm{d} \mathcal{S} \\
=\left(\frac{4 \pi \mathrm{i} P o \sin \alpha_{p}}{5}\right)\left(2-\mathcal{E}^{2}\right) \sqrt{1-\mathcal{E}^{2}}
\end{array}
$$

where, upon using (3.5)-(3.7) with $n=1, k=0$ and $\sigma_{110}=1 /\left(2-\mathcal{E}^{2}\right)$, we have

$$
\int_{\mathcal{V}}\left|\mathbf{u}_{110}\right|^{2} \mathrm{~d} \mathcal{V}=\frac{3 \pi\left(1-\mathcal{E}^{2}\right)^{3}}{10 \sqrt{1-\mathcal{E}^{2}}}
$$

It is worth mentioning that the boundary-layer flux $(\hat{\mathbf{n}} \cdot \widehat{\mathbf{u}})$ on the left-hand side of (3.13) is a function of the unknown coefficient $\mathcal{A}_{110}$ in connection with the viscous boundary layer $\widetilde{\mathbf{u}}$.

\subsection{Asymptotic solution}

Since the boundary layer flow $\widetilde{\mathbf{u}}$ on the bounding surface $\mathcal{S}$, which produces the flux $(\hat{\mathbf{n}} \cdot \widehat{\mathbf{u}})_{\mathcal{S}}$ in $(3.13)$, is required, we now adopt spheroidal polar coordinates $(\eta, \tau, \phi)$ which 
is mathematically more convenient for performing the boundary-layer analysis in an oblate spheroid of arbitrary eccentricity. In oblate spheroidal coordinates, the inertial mode with $m=1$ needed in the solvability condition (3.13) is of the form

$$
\begin{aligned}
p_{110}(\eta, \tau, \phi) & =\frac{3}{2}\left(\eta^{2}+\mathcal{E}^{2}\right)^{1 / 2}\left(1-\tau^{2}\right)^{1 / 2} \eta \tau \mathrm{e}^{\mathrm{i} \phi}, \\
\hat{\boldsymbol{\eta}} \cdot \mathbf{u}_{110}(\eta, \tau, \phi) & =\mathrm{i}\left[\frac{3\left(2-\mathcal{E}^{2}\right)}{4\left(1-\mathcal{E}^{2}\right)}\right] \frac{\tau \mathcal{E}^{2} \sqrt{1-\tau^{2}}\left(1-\mathcal{E}^{2}-\eta^{2}\right)}{\sqrt{\eta^{2}+\tau^{2} \mathcal{E}^{2}}} \mathrm{e}^{\mathrm{i} \phi}, \\
\hat{\boldsymbol{\phi}} \cdot \mathbf{u}_{110}(\eta, \tau, \phi) & =\left[\frac{3\left(2-\mathcal{E}^{2}\right)}{4\left(1-\mathcal{E}^{2}\right)}\right] \eta \tau \mathrm{e}^{\mathrm{i} \phi}, \\
\hat{\boldsymbol{\tau}} \cdot \mathbf{u}_{110}(\eta, \tau, \phi) & =\mathrm{i}\left[\frac{3\left(2-\mathcal{E}^{2}\right)}{4\left(1-\mathcal{E}^{2}\right)}\right] \frac{\eta \sqrt{\eta^{2}+\mathcal{E}^{2}}\left(1-\mathcal{E}^{2}+\tau^{2} \mathcal{E}^{2}\right)}{\sqrt{\eta^{2}+\tau^{2} \mathcal{E}^{2}}} \mathrm{e}^{\mathrm{i} \phi},
\end{aligned}
$$

which is valid for an oblate spheroid with $0<\mathcal{E}<1$.

The viscous boundary-layer flow $(\widetilde{\mathbf{u}}, \widetilde{p})$ on the bounding surface $\mathcal{S}$ in the asymptotic expansions (3.1) and (3.2) is described by

$$
\mathrm{i} \widetilde{\mathbf{u}}+2 \hat{\mathbf{z}} \times \widetilde{\mathbf{u}}+\hat{\mathbf{n}}(\hat{\mathbf{n}} \cdot \nabla \widetilde{p})=\frac{\partial^{2} \widetilde{\mathbf{u}}}{\partial \xi^{2}},
$$

where a boundary-layer stretched coordinate,

$$
\xi=\frac{\left[\left(1-\mathcal{E}^{2}\right)^{1 / 2}-\eta\right]}{\sqrt{E k}},
$$

is introduced: $\xi=0$ at the bounding surface $\mathcal{S}$ while $\xi \rightarrow \infty$ defines the edge of the thin viscous boundary layer for $0<E k \ll 1$. Decompose the boundary flow $\widetilde{\mathbf{u}}$ into the tangential and normal components,

$$
\widetilde{\mathbf{u}}=\widetilde{\mathbf{u}}_{\text {tang }}+\hat{\mathbf{n}}(\hat{\mathbf{n}} \cdot \widetilde{\mathbf{u}}) .
$$

By applying the operators $\hat{\mathbf{n}} \times$ and $\hat{\mathbf{n}} \times \hat{\mathbf{n}} \times$ onto the above second-order differential equation and, then, combining the two resulting equations to form a single equation for the tangential component $\widetilde{\mathbf{u}}_{t a n g}$ of $\widetilde{\mathbf{u}}$, we obtain a fourth-order differential equation

$$
\left(\frac{\partial^{2}}{\partial \xi^{2}}-\mathrm{i}\right)^{2} \widetilde{\mathbf{u}}_{t a n g}+\left(\frac{2 \tau}{\sqrt{1-\mathcal{E}^{2}+\mathcal{E}^{2} \tau^{2}}}\right)^{2} \widetilde{\mathbf{u}}_{t a n g}=0
$$

subject to the four boundary conditions

$$
\begin{aligned}
& \left(\widetilde{\mathbf{u}}_{\text {tang }}\right)_{\xi=0}=-\frac{3\left(2-\mathcal{E}^{2}\right) \mathcal{A}_{110}}{4\left(1-\mathcal{E}^{2}\right)^{1 / 2}}\left[\mathrm{i}\left(1-\mathcal{E}^{2}+\tau^{2} \mathcal{E}^{2}\right)^{1 / 2} \hat{\boldsymbol{\tau}}+\tau \hat{\boldsymbol{\phi}}\right] \mathrm{e}^{\mathrm{i} \phi}, \\
& \left(\widetilde{\mathbf{u}}_{\text {tang }}\right)_{\xi=\infty}=0, \\
& \left(\frac{\partial^{2} \widetilde{\mathbf{u}}_{\text {tang }}}{\partial \xi^{2}}\right)_{\xi=0}=\frac{3\left(2-\mathcal{E}^{2}\right) \mathcal{A}_{110}}{4\left(1-\mathcal{E}^{2}\right)^{1 / 2}} \\
& \times\left\{\left[\left(1-\mathcal{E}^{2}+\tau^{2} \mathcal{E}^{2}\right)^{1 / 2}-\frac{2 \tau^{2}}{\left(1-\mathcal{E}^{2}+\tau^{2} \mathcal{E}^{2}\right)^{1 / 2}}\right] \hat{\boldsymbol{\tau}}+\mathrm{i} \tau \hat{\boldsymbol{\phi}}\right\} \mathrm{e}^{\mathrm{i} \phi}, \\
& \left(\frac{\partial^{2} \widetilde{\mathbf{u}}_{\text {tang }}}{\partial \xi^{2}}\right)_{\xi=\infty}=0 .
\end{aligned}
$$

A straightforward analysis shows that the tangential component $\widetilde{\mathbf{u}}_{\text {tang }}$ of $\widetilde{\mathbf{u}}$ satisfying 


$\begin{array}{lllll}\mathcal{E} & \left(\mathcal{I}_{r}\right)_{\text {num }} & \left(\mathcal{I}_{r}\right)_{\text {asym }} & \left(\mathcal{I}_{i}\right)_{\text {num }} & \left(\mathcal{I}_{i}\right)_{\text {asym }} \\ 0.01 & -0.1949 & -0.1949 & -1.9763 & -1.9763 \\ 0.05 & -0.1937 & -0.1937 & -1.9729 & -1.9724 \\ 0.1 & -0.1899 & -0.1899 & -1.9622 & -1.9603 \\ 0.2 & -0.1755 & -0.1748 & -1.9189 & -1.9117 \\ 0.3 & -0.1535 & -0.1495 & -1.8448 & -1.8306 \\ 0.4 & -0.1273 & -0.1142 & -1.7375 & -1.7171\end{array}$

TABLE 1. Several numerical (with the subscript num and computed from numerical integration) and asymptotic (with the subscript asym and computed from the corresponding asymptotic expression) values for $\mathcal{I}_{r}$ and $\mathcal{I}_{i}$ with different values of $\mathcal{E}$.

both (3.18) and the four conditions is

$$
\begin{aligned}
\widetilde{\mathbf{u}}_{\text {tang }}=\frac{\mathrm{i} 3\left(2-\mathcal{E}^{2}\right) \mathcal{A}_{110}}{8\left(1-\mathcal{E}^{2}\right)^{1 / 2}} & {\left[\left(\tau-\sqrt{1-\mathcal{E}^{2}+\mathcal{E}^{2} \tau^{2}}\right)(\hat{\boldsymbol{\tau}}+\mathrm{i} \hat{\boldsymbol{\phi}}) \mathrm{e}^{\gamma^{+} \xi}\right.} \\
+ & \left.\left(\tau+\sqrt{1-\mathcal{E}^{2}+\mathcal{E}^{2} \tau^{2}}\right)(-\hat{\boldsymbol{\tau}}+\mathrm{i} \hat{\boldsymbol{\phi}}) \mathrm{e}^{\gamma^{-} \xi}\right] \mathrm{e}^{\mathrm{i} \phi},
\end{aligned}
$$

where $\gamma^{ \pm}$is a function of $\tau$ and $\mathcal{E}$

$$
\begin{aligned}
& \gamma^{+}=-\frac{\sqrt{2}}{2}\left[1+\frac{i\left(\sqrt{1-\mathcal{E}^{2}+\mathcal{E}^{2} \tau^{2}}+2 \tau\right)}{\left|\sqrt{1-\mathcal{E}^{2}+\mathcal{E}^{2} \tau^{2}}+2 \tau\right|}\right] \frac{\left|\sqrt{1-\mathcal{E}^{2}+\mathcal{E}^{2} \tau^{2}}+2 \tau\right|^{1 / 2}}{\left(1-\mathcal{E}^{2}+\mathcal{E}^{2} \tau^{2}\right)^{1 / 4}}, \\
& \gamma^{-}=-\frac{\sqrt{2}}{2}\left[1+\frac{\mathrm{i}\left(\sqrt{1-\mathcal{E}^{2}+\mathcal{E}^{2} \tau^{2}}-2 \tau\right)}{\left|\sqrt{1-\mathcal{E}^{2}+\mathcal{E}^{2} \tau^{2}}-2 \tau\right|}\right] \frac{\left|\sqrt{1-\mathcal{E}^{2}+\mathcal{E}^{2} \tau^{2}}-2 \tau\right|^{1 / 2}}{\left(1-\mathcal{E}^{2}+\mathcal{E}^{2} \tau^{2}\right)^{1 / 4}}
\end{aligned}
$$

Evidently, the viscous boundary layer breaks down at the critical spheroidal latitudes $\tau_{c}= \pm\left[\left(1-\mathcal{E}^{2}\right) /\left(4-\mathcal{E}^{2}\right)\right]^{1 / 2}$, where the thickness of the boundary layer may change from $\mathrm{O}\left(E k^{1 / 2}\right)$ to $\mathrm{O}\left(E k^{2 / 5}\right)$ (Roberts and Stewartson 1965). However, this is unlikely to significantly affect the leading-order solution at an asymptotically small $E k$ because the total influx from the singular region is small (Roberts and Stewartson 1965; Busse 1968; Hollerbach and Kerswell 1995; Tilgner and Busse 2001).

With the availability of the tangential component of $\widetilde{\mathbf{u}}$, the mass flux at the outer edge of the boundary layer $(\hat{\mathbf{n}} \cdot \widehat{\mathbf{u}})_{\mathcal{S}}$ in $(3.13)$, which links the boundary-layer solution to the secondary interior flow, can derived from the boundary-layer mass conservation:

$$
\begin{aligned}
(\hat{\mathbf{n}} \cdot \widehat{\mathbf{u}})_{\mathcal{S}} & =\frac{\sqrt{E k}}{\sqrt{1-\mathcal{E}^{2}+\mathcal{E}^{2} \tau^{2}}} \int_{0}^{\infty}\left\{\left[\frac{\mathrm{i}\left(1-\mathcal{E}^{2}+\mathcal{E}^{2} \tau^{2}\right)}{\left(1-\tau^{2}\right)^{1 / 2}} \hat{\boldsymbol{\phi}} \cdot \widetilde{\mathbf{u}}_{\text {tang }}\right]\right. \\
& \left.+\frac{\partial}{\partial \tau}\left[\sqrt{\left(1-\mathcal{E}^{2}+\mathcal{E}^{2} \tau^{2}\right)\left(1-\tau^{2}\right)} \hat{\boldsymbol{\tau}} \cdot \widetilde{\mathbf{u}}_{t a n g}\right]\right\} \mathrm{e}^{\mathrm{i} \phi} \mathrm{d} \xi
\end{aligned}
$$

This expression is needed for evaluating the surface integral in the solvability condition (3.13) which determines the amplitude $\mathcal{A}_{110}$ :

$$
\begin{aligned}
-\frac{\mathrm{i} 3 \pi \mathcal{A}_{110} \mathcal{E}^{2}\left(2-\mathcal{E}^{2}\right)^{2}}{10\left(1-\mathcal{E}^{2}\right)^{1 / 2}}+\frac{9 \mathrm{i} \pi\left(2-\mathcal{E}^{2}\right) \sqrt{E k} \mathcal{A}_{110}}{4 \sqrt{2}}\left(\mathcal{I}_{r}+\mathrm{i} \mathcal{I}_{i}\right) \\
=\frac{\mathrm{i} 4 \pi P o \sin \alpha_{p}}{5}\left(2-\mathcal{E}^{2}\right)\left(1-\mathcal{E}^{2}\right)^{1 / 2}
\end{aligned}
$$


where $\mathcal{I}_{r}$ and $\mathcal{I}_{i}$ denote the two integrals

$$
\begin{aligned}
\mathcal{I}_{r} & =\int_{-1}^{+1}\left\{\left(1-\mathcal{E}^{2}+\mathcal{E}^{2} \tau^{2}\right)^{3 / 4}\right. \\
& \left.\times\left[\frac{\left(-\tau+\sqrt{1-\mathcal{E}^{2}+\mathcal{E}^{2} \tau^{2}}\right)\left(1-2 \tau^{2}+\tau \sqrt{1-\mathcal{E}^{2}+\mathcal{E}^{2} \tau^{2}}\right)}{\left|2 \tau+\sqrt{1-\mathcal{E}^{2}+\mathcal{E}^{2} \tau^{2}}\right|^{1 / 2}}\right]\right\} \mathrm{d} \tau \\
\mathcal{I}_{i} & =\int_{-1}^{+1}\left\{\left(1-2 \tau^{2}+\tau \sqrt{1-\mathcal{E}^{2}+\mathcal{E}^{2} \tau^{2}}\right)\right. \\
& \left.\times \frac{\left(2 \tau+\sqrt{1-\mathcal{E}^{2}+\mathcal{E}^{2} \tau^{2}}\right)\left(\tau-\sqrt{1-\mathcal{E}^{2}+\mathcal{E}^{2} \tau^{2}}\right)\left(1-\mathcal{E}^{2}+\mathcal{E}^{2} \tau^{2}\right)^{3 / 4}}{\left|\tau+\sqrt{1-\mathcal{E}^{2}+\mathcal{E}^{2} \tau^{2}}\right|^{3 / 2}}\right\} \mathrm{d} \tau .
\end{aligned}
$$

Solving (3.21) for $\mathcal{A}_{110}$ yields

$$
\mathcal{A}_{110}=\frac{16 \sqrt{2} \text { Po } \sin \alpha_{p}\left(1-\mathcal{E}^{2}\right)}{45 \sqrt{E k}\left(\mathcal{I}_{r}+\mathrm{i} \mathcal{I}_{i}\right) \sqrt{1-\mathcal{E}^{2}}-6 \sqrt{2}\left(2-\mathcal{E}^{2}\right) \mathcal{E}^{2}} .
$$

In the expansion (3.1), setting $\mathcal{A}_{m n k}=0$ for $m \neq 1$ and $\mathcal{A}_{1 n k}=0$ for $n \neq 1$ and $k \neq 0$ and inserting the expressions for $\mathcal{A}_{110}, \mathbf{u}_{110}$ and $\widetilde{\mathbf{u}}_{\text {tang }}$ result in the leading-order asymptotic solution for the weakly precessing flow

$$
\begin{aligned}
& \mathbf{u}(\eta, \tau, \phi, t)=\left[\frac{\mathrm{i} 4 \sqrt{2} P o \sin \alpha_{p}\left(2-\mathcal{E}^{2}\right)}{15 \sqrt{E k}\left(\mathcal{I}_{r}+\mathrm{i} \mathcal{I}_{i}\right) \sqrt{1-\mathcal{E}^{2}}-2 \sqrt{2} \mathcal{E}^{2}\left(2-\mathcal{E}^{2}\right)}\right] \\
& \times\left\{\left[\frac{\tau \mathcal{E}^{2} \sqrt{\left(1-\tau^{2}\right)}\left(1-\mathcal{E}^{2}-\eta^{2}\right)}{\sqrt{\eta^{2}+\tau^{2} \mathcal{E}^{2}}} \hat{\boldsymbol{\eta}}-\mathrm{i}(\eta \tau) \hat{\boldsymbol{\phi}}+\frac{\eta \sqrt{\eta^{2}+\mathcal{E}^{2}}\left(1-\mathcal{E}^{2}+\tau^{2} \mathcal{E}^{2}\right)}{\sqrt{\eta^{2}+\tau^{2} \mathcal{E}^{2}}} \hat{\boldsymbol{\tau}}\right]\right. \\
& +\frac{\left(1-\mathcal{E}^{2}\right)^{1 / 2}}{2}\left[\left(\tau-\sqrt{1-\mathcal{E}^{2}+\mathcal{E}^{2} \tau^{2}}\right)(\hat{\boldsymbol{\tau}}+\mathrm{i} \hat{\boldsymbol{\phi}}) \mathrm{e}^{\gamma^{+} \xi}\right. \\
& \left.\left.+\left(\tau+\sqrt{1-\mathcal{E}^{2}+\mathcal{E}^{2} \tau^{2}}\right)(-\hat{\boldsymbol{\tau}}+\mathrm{i} \hat{\boldsymbol{\phi}}) \mathrm{e}^{\gamma^{-} \xi}\right]\right\} \mathrm{e}^{\mathrm{i}(\phi+t)},
\end{aligned}
$$

which satisfies the no-slip boundary condition (2.4) and is valid for an oblate spheroid of arbitrary eccentricity with $0<\mathcal{E}<1$ and arbitrary precession angle with $0<\alpha_{p}<\pi / 2$. The corresponding kinetic energy density $E_{\text {kin }}$ neglecting the small contribution from the viscous boundary layer is

$$
\begin{aligned}
E_{\text {kin }} & =\frac{1}{2 \mathcal{V}} \int_{\mathcal{V}}|\mathbf{u}|^{2} \mathrm{~d} \mathcal{V} \\
= & \frac{144\left(P o \sin \alpha_{p}\right)^{2}\left(1-\mathcal{E}^{2}\right)\left(2-\mathcal{E}^{2}\right)^{3}}{5\left\{\left[45 \mathcal{I}_{r} \sqrt{E k\left(1-\mathcal{E}^{2}\right)}-6 \sqrt{2} \mathcal{E}^{2}\left(2-\mathcal{E}^{2}\right)\right]^{2}+2025\left(1-\mathcal{E}^{2}\right) \mathcal{I}_{i}^{2} E k\right\}},
\end{aligned}
$$

which measures the typical amplitude of a precessing flow and can be used to compare with the result of direct numerical simulation.

Though both the classical solution of Poincaré (1910) (the Poincaré flow) and our asymptotic solution (3.23) are valid for a spheroid of arbitrary eccentricity, there are at least four essential differences between them: (i) (3.23) satisfies the non-slip boundary condition while the Poincaré solution obeys only the inviscid condition; (ii) the amplitude of the Poincaré flow depends on $P_{o}$ and $\mathcal{E}$ but the amplitude of (3.23) is a function of $E k, P o$ and $\mathcal{E}$; (iii) the limit $\mathcal{E} \rightarrow 0$ in the Poincaré solution is singular while (3.23) is valid for $0 \leqslant \mathcal{E}<1$ and, moreover, the limit $\mathcal{E} \rightarrow 0$ gives rise to the spherical precessing flow (Zhang et al. 2010); (iv) the Poincaré solution is, since the spheroidal container 
is viscously uncoupled from the fluid, non-unique (Wu and Roberts 2009) while this non-uniqueness is removed by the effect of viscosity in (3.23).

Unfortunately, both the integrals, $\mathcal{I}_{r}$ and $\mathcal{I}_{i}$ in the expressions (3.23) and (3.24), have to be evaluated, in general, via a numerical method. When $\mathcal{E}$ is moderately small, however, an asymptotic expression for $\mathcal{I}_{r}$ or $\mathcal{I}_{i}$ can be obtained. Note that a careful manipulation is required in evaluating $\mathcal{I}_{r}$ and $\mathcal{I}_{i}$ because there exist two critical values of $\tau_{c}$ at which the boundary-layer solution breaks down. For an oblate spheroid with small $\mathcal{E}$, the integrals $\mathcal{I}_{r}$ and $\mathcal{I}_{i}$ can be evaluated approximately on the basis of expansion in $\mathcal{E}^{2}$, which gives

$$
\begin{aligned}
& \mathcal{I}_{r}=-\frac{2}{35}(19-9 \sqrt{3})+\mathcal{E}^{2}\left[\frac{2}{385}\left(\frac{1669}{9}-51 \sqrt{3}\right)\right]+\mathrm{O}\left(\mathcal{E}^{4}\right), \\
& \mathcal{I}_{i}=-\frac{2}{35}(19+9 \sqrt{3})+\mathcal{E}^{2}\left[\frac{4}{77}\left(\frac{47}{9}+15 \sqrt{3}\right)\right]+\mathrm{O}\left(\mathcal{E}^{4}\right) .
\end{aligned}
$$

These provide a reasonably accurate approximation to $\mathcal{I}_{r}$ and $\mathcal{I}_{i}$ for moderate small values of $\mathcal{E}$. For example, the numerical integration at $\mathcal{E}=0.25$ gives $\left(\mathcal{I}_{r}\right)_{\text {num }}=-0.1653$ while the asymptotic formula yields $\left(\mathcal{I}_{r}\right)_{\text {asym }}=-0.1634$; the numerical integration of $\mathcal{I}_{i}$ at $\mathcal{E}=0.25$ gives $\left(\mathcal{I}_{i}\right)_{\text {num }}=-1.886$ while the asymptotic formula produces $\left(\mathcal{I}_{i}\right)_{\text {asym }}=$ -1.875 . More examples of both the numerical and asymptotic values for $\mathcal{I}_{r}$ and $\mathcal{I}_{i}$ are listed in Table 1. Integrability of the two singular integrals $\mathcal{I}_{r}$ and $\mathcal{I}_{i}$ in a way reflects the fact that the total influx from the singular regions of the boundary layer is small.

With the above expressions for $\mathcal{I}_{r}$ and $\mathcal{I}_{i}$, the asymptotic solution of a weakly precessing flow in slightly flattened oblate spheroids can be written in the explicitly analytical form

$$
\begin{aligned}
\mathbf{u} & =\frac{\mathrm{i} 4 \sqrt{2} P o \sin \alpha_{p}\left(2-\mathcal{E}^{2}\right)}{15 \sqrt{E k} \sqrt{1-\mathcal{E}^{2}}\left[\left(-0.195+0.504 \mathcal{E}^{2}\right)+\mathrm{i}\left(-1.98+1.62 \mathcal{E}^{2}\right)\right]-2 \sqrt{2} \mathcal{E}^{2}\left(2-\mathcal{E}^{2}\right)} \\
& \times\left\{\left[\frac{\tau \mathcal{E}^{2} \sqrt{\left(1-\tau^{2}\right)}\left(1-\mathcal{E}^{2}-\eta^{2}\right)}{\sqrt{\eta^{2}+\tau^{2} \mathcal{E}^{2}}} \hat{\boldsymbol{\eta}}-\mathrm{i}(\eta \tau) \hat{\boldsymbol{\phi}}+\frac{\mathrm{i} \eta \sqrt{\eta^{2}+\mathcal{E}^{2}}\left(1-\mathcal{E}^{2}+\tau^{2} \mathcal{E}^{2}\right)}{\sqrt{\eta^{2}+\tau^{2} \mathcal{E}^{2}}} \hat{\boldsymbol{\tau}}\right]\right. \\
& +\frac{\left(1-\mathcal{E}^{2}\right)^{1 / 2}}{2}\left[\left(\tau-\sqrt{1-\mathcal{E}^{2}+\mathcal{E}^{2} \tau^{2}}\right)(\hat{\boldsymbol{\tau}}+\mathrm{i} \hat{\boldsymbol{\phi}}) \mathrm{e}^{\gamma^{+} \xi}\right. \\
& \left.\left.+\left(\tau+\sqrt{1-\mathcal{E}^{2}+\mathcal{E}^{2} \tau^{2}}\right)(-\hat{\boldsymbol{\tau}}+\mathrm{i} \hat{\boldsymbol{\phi}}) \mathrm{e}^{\gamma^{-} \xi}\right]\right\} \mathrm{e}^{\mathrm{i}(\phi+t)} .
\end{aligned}
$$

The corresponding kinetic energy density $E_{\text {kin }}$ becomes

$$
\begin{gathered}
E_{\text {kin }}=\frac{144\left(P o \sin \alpha_{p}\right)^{2}\left(1-\mathcal{E}^{2}\right)\left(2-\mathcal{E}^{2}\right)^{3}}{5}\left\{\left[\left(-88.942+72.942 \mathcal{E}^{2}\right) \sqrt{\left(1-\mathcal{E}^{2}\right) E k}\right]^{2}\right. \\
\left.+\left[\left(-8.7725+22.701 \mathcal{E}^{2}\right) \sqrt{E k\left(1-\mathcal{E}^{2}\right)}-6 \sqrt{2} \mathcal{E}^{2}\left(2-\mathcal{E}^{2}\right)\right]^{2}\right\}^{-1}
\end{gathered}
$$

valid for an oblate spheroid with a moderately small value of $\mathcal{E}$ (see Table 1). A possible transformation between the asymptotic solution of Busse (1968) obtained in the precession frame for $\mathcal{E} \ll 1$ to our asymptotic solution (3.25) in the mantle frame is discussed in $\S 5$.

\section{Direct numerical simulation}

The primary objectives of direct numerical simulation, which is not restricted by $0<$ $|P o| \ll 1$ and $0<E k \ll 1$, are twofold: (i) to validate the asymptotic solutions (3.23) and (3.24) that are valid only for the weakly precessing flow at $E k \ll 1$ and (ii) to reveal the key characteristics of the strongly precessing flow for an oblate spheroid of arbitrary 


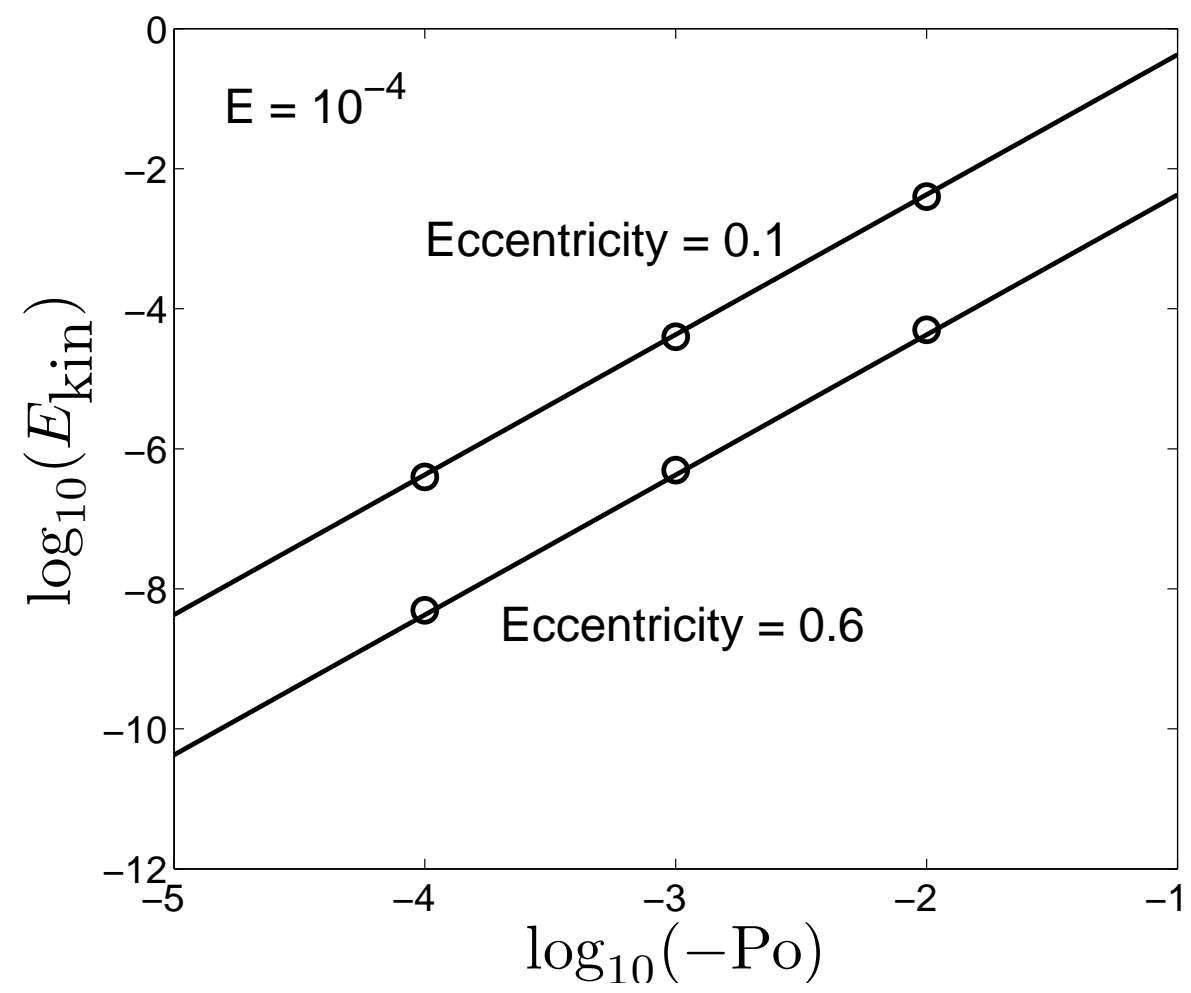

Figure 2. Kinetic energies, $E_{\text {kin }}$, of the precessing flows for $E k=10^{-4}$ plotted as a function of $|P o|$ at two different values of $\mathcal{E}(\mathcal{E}=0.1$ and $\mathcal{E}=0.6)$. The solid lines represent the asymptotic solutions computed from the expression (3.24) while the circles show the results obtained from fully nonlinear numerical simulation.

eccentricity. Nonlinear numerical simulation concentrates on the cases with $0<E k \ll 1$ and moderate values of $|P o|$ such that direct comparison between the asymptotic and numerical solutions can be readily made and nonlinear effects on the precessing flow can be clearly illustrated.

Local numerical methods like finite element methods are particularly suitable for an oblate spheroid of arbitrary eccentricity where the standard spectral method would be obviously inconvenient. For the sake of completeness, we shall briefly discuss the finite element method which is employed in simulating precession-driven flow in spheroidal cavities; the details of the numerical method can be found in Chan et al. (2010). A threedimensional tetrahedralization of the spheroidal cavity produces a finite element mesh that does not have pole or central numerical singularities, and the three-dimensional mesh is flexible enough to construct not only an oblate spheroid of arbitrary eccentricity but also more nodes in the vicinity of its bounding surface in order to resolve the thin viscous boundary layer. A sketch of the finite element mesh for a spheroidal cavity with $\mathcal{E}=0.5$ is illustrated in Fig. 1. In our numerical simulation, we typically use a finite element mesh of nearly $10^{6}$ nodes with about $3 \times 10^{6}$ unknowns. A mixed finite element of HoodTaylor type is adopted: in each tetrahedral element, a piecewise quadratic polynomial is employed to approximate the velocity $\mathbf{u}$ while a piecewise linear polynomial is used to approximate the pressure $p$.

After making the tetrahedralization of the whole spheroidal cavity, we construct the temporal discretization of the numerical precession model. Let $T_{f}$ be a fixed final time 


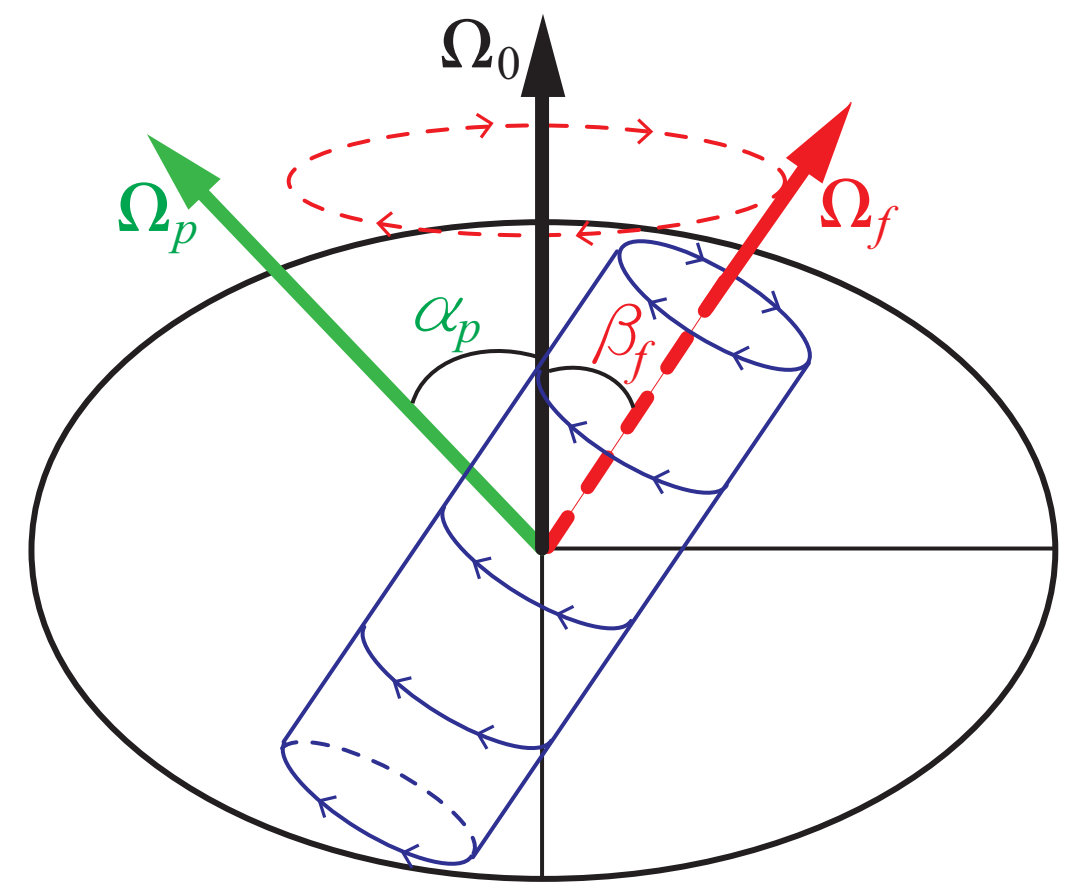

FIGURE 3. Sketch of the three vectors describing a precessing flow in the mantle frame: $\boldsymbol{\Omega}_{0}$ is at the symmetry axis and independent of $t, \boldsymbol{\Omega}_{p}$ is the time-dependent precession vector inclined at an angle $\alpha_{p}\left(0<\alpha_{p} \leqslant \pi / 2\right)$ to $\boldsymbol{\Omega}_{0}$ described by the expression (2.3), and $\boldsymbol{\Omega}_{f}$ represents the rotation vector of fluid motion that moves retrogradely on a cone (indicated by the dashed circle) inclined at an angle $\beta_{f}\left(0<\beta_{f} \leqslant \pi / 2\right)$ to $\boldsymbol{\Omega}_{0}$.

of a numerical simulation. We divide the time interval $\left[0, T_{f}\right]$ into $M$ equally spaced subintervals using the following nodal points

$$
0=t_{0}<t_{1}<t_{2}<\ldots<t_{M}=T_{f},
$$

where $t_{n}=n \Delta t$ for $n=0,1, \ldots, M$. Let $\mathbf{u}(\mathbf{r}, t)$ be a function continuous with respect to $t$. We shall denote $\mathbf{u}^{n}(\mathbf{r})=\mathbf{u}\left(\mathbf{r}, t_{n}\right)$ for $n=0,1, \ldots, M$. An implicit time stepping scheme is then employed for the time advancement of integration in which we adopt a second-order backward differentiation formula for the time derivative

$$
\left(\frac{\partial \mathbf{u}}{\partial t}\right)^{n+1}=\frac{3 \mathbf{u}^{n+1}-4 \mathbf{u}^{n}+\mathbf{u}^{n-1}}{2 \Delta t}+O\left(\Delta t^{2}\right)
$$

while the nonlinear term $\mathbf{u} \cdot \nabla \mathbf{u}$ at $t=t_{n+1}$ is approximated by the implicit formula

$$
\mathbf{u}^{n+1} \cdot \nabla \mathbf{u}^{n+1}=\left(2 \mathbf{u}^{n}-\mathbf{u}^{n-1}\right) \cdot \nabla \mathbf{u}^{n+1}+O\left(\Delta t^{2}\right) .
$$

An implicit temporal discretization of the full equations (2.1)-(2.2) produces

$$
\begin{gathered}
\frac{3 \mathbf{u}^{n+1}-4 \mathbf{u}^{n}+\mathbf{u}^{n-1}}{2 \Delta t}+\left(2 \mathbf{u}^{n}-\mathbf{u}^{n-1}\right) \cdot \nabla \mathbf{u}^{n+1}+2\left(\hat{\mathbf{z}}+\widehat{\boldsymbol{\Omega}}_{p}^{n+1}\right) \times \mathbf{u}^{n+1} \\
=-\nabla P^{n+1}+E \nabla^{2} \mathbf{u}^{n+1}+P o\left[\mathbf{r} \times\left(\widehat{\boldsymbol{\Omega}}_{p}^{n+1} \times \hat{\mathbf{z}}\right)\right], \\
\nabla \cdot \mathbf{u}^{n+1}=0,
\end{gathered}
$$

which are solved, starting from an arbitrary initial condition, to find $\mathbf{u}^{n+1}, P^{n+1}$ for given $\mathbf{u}^{n}$ and $P^{n}$ on modern parallel computers. Note that the velocity $\mathbf{u}^{n+1}$ and pressure $P^{n+1}$ 


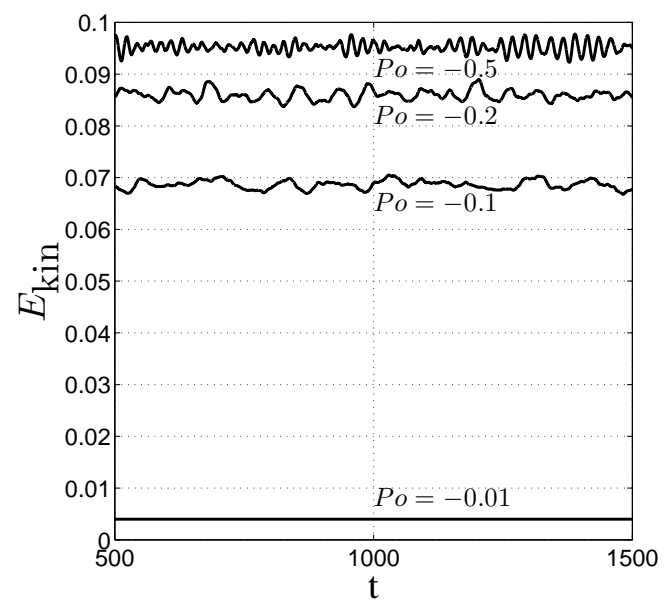

(a)

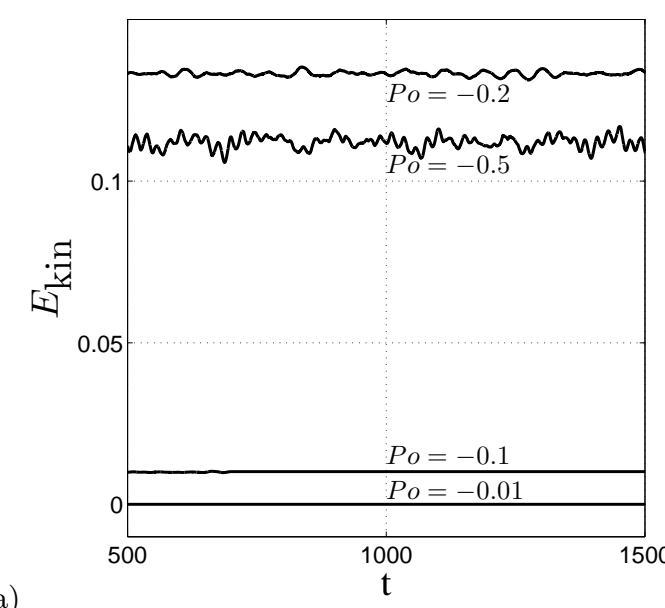

(b)

FiguRE 4 . Kinetic energy $E_{\text {kin }}$ of the precessionally driven flows for several different values of Po plotted as a function of $t$ : (a) for $\mathcal{E}=0.1, E=10^{-4}$ at $\alpha_{p}=23.5^{\circ}$ and (b) for $\mathcal{E}=0.6$, $E=10^{-4}$ at $\alpha_{p}=23.5^{\circ}$.

are solved together as a saddle point problem (Chan et al. 2010). Although a fixed timestep approach is adopted in the current precession model, a variable time-step scheme can be readily implemented. The implicit scheme allows us to use a relatively large timestep $\Delta t$ ranging from $10^{-3}$ to $10^{-1}$ in our simulation. No spatial symmetries with respect to the equator or a meridian plane are imposed on our fully three-dimensional nonlinear numerical simulation. It is worth mentioning that our simulation becomes quite expensive when both $E k$ and $\mathcal{E}$ are small such that the viscous boundary layer plays an active role: it usually takes about $\mathrm{O}\left(E k^{-1 / 2}\right)$ dimensionless time units to reach the nonlinear equilibrium state of a precessing flow starting from an arbitrary initial condition. In a way, very expensive computation at small $E k$ and $\mathcal{E}$ signals that the coupling between the spheroidal container and the viscous fluid becomes viscously predominant.

A satisfactory quantitative agreement, when $E k \leqslant 10^{-4}$, is achieved between the analytical expressions (3.23)-(3.24) and nonlinear direct numerical simulation for $0<\mathcal{E}<1$ when $|P o| \ll 1$. Both the asymptotic expression (3.24) and the result of nonlinear numerical simulation at $E=10^{-4}$ are presented in Figure 2, showing the kinetic energies $E_{\text {kin }}$ of the precessing flows as a function of $|P o|$ at two different values of eccentricity, $\mathcal{E}=0.1$ and $\mathcal{E}=0.6$. It is shown that the asymptotic solution provides an accurate approximation for the precessing flow in an oblate spheroid of arbitrary eccentricity when $|P o| \ll 1$ and $E k \ll 1$.

An important question is how to illustrate, and thus to help understand, the spatial structure of a time-dependent, nonlinear numerical solution in the mantle frame of reference. It is found that the primary features of a precessing flow in an oblate spheroid are largely characterized by the three vectors - the basic rotation vector $\boldsymbol{\Omega}_{0}$, the precession vector $\boldsymbol{\Omega}_{p}$ and the rotation vector $\boldsymbol{\Omega}_{f}$ of fluid motion - which are depicted in Figure 3 . The direction of $\boldsymbol{\Omega}_{0}$ is fixed at the symmetry axis and independent of $t$ in the mantle frame, the direction of $\boldsymbol{\Omega}_{p}$ inclined at an angle $\alpha_{p}\left(0<\alpha_{p} \leqslant \pi / 2\right)$ to $\boldsymbol{\Omega}_{0}$ varies with $t$ in the way described by the expression (2.3), and the direction of $\boldsymbol{\Omega}_{f}$ inclined at an angle $\beta_{f}\left(0<\beta_{f} \leqslant \pi / 2\right)$ to $\Omega_{0}$ is time-dependent and moves retrogradely on a cone (which is indicated by the dashed circle attached to the vector $\boldsymbol{\Omega}_{f}$ in Figure 3 ) with an angle $\beta_{f}$ to $\boldsymbol{\Omega}_{0}$. Our discussion will center on the size of the angle $\beta_{f}$ and its connection with an azimuthal mean flow. 

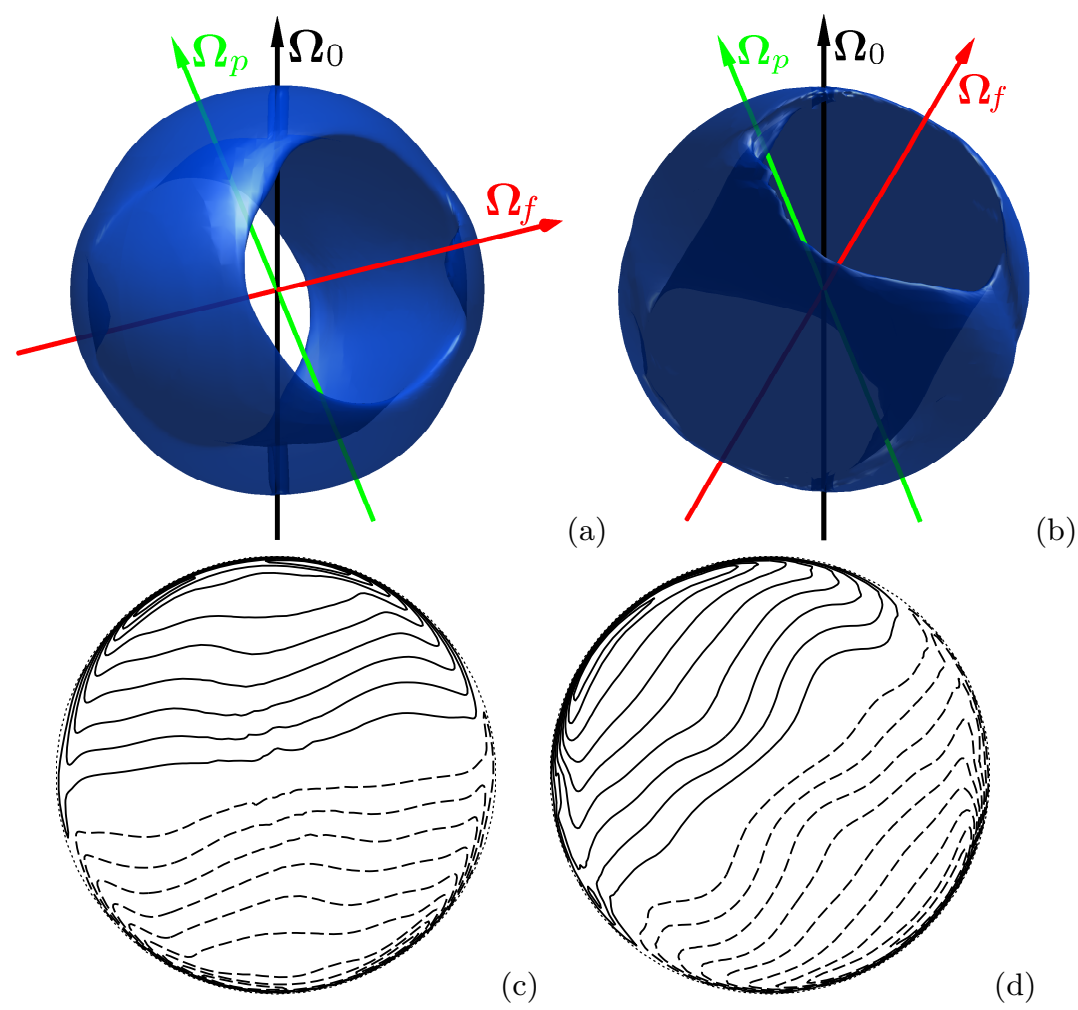

(c)

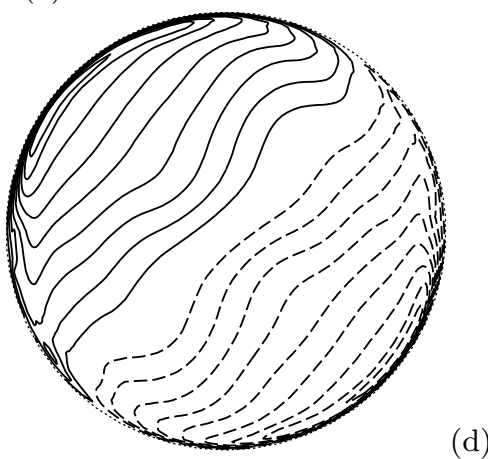

FIgURE 5. Isosurface of the azimuthal flow together with the position of the three vectors $\boldsymbol{\Omega}_{0}, \boldsymbol{\Omega}_{p}$ and $\boldsymbol{\Omega}_{f}$ - obtained with $\mathcal{E}=0.1, E=10^{-4}$ and $\alpha_{p}=23.5^{\circ}$ : (a) for Po $=-0.01$ with $\beta_{f}=80^{\circ}$ and (b) for $P o=-0.2$ with $\beta_{f}=46^{\circ}$. The corresponding contours of $\hat{\phi} \cdot \mathbf{u}$ in a meridional plane: (c) for $P o=-0.01$ and (d) for $P o=-0.2$. Blue colour of the isosurface in (a,b) indicates retrogradely azimuthal flow with respect to the vector $\boldsymbol{\Omega}_{f}$. In $(\mathrm{c}, \mathrm{d})$, solid contours denote flow into the paper while dashed contours are for flow out of the paper.

For a time-dependent numerical solution obtained in the mantle frame of reference, the size of the angle $\beta_{f}$ can be approximately determined by transforming the numerical solutions at various instants from the computational coordinate system based on the vector $\boldsymbol{\Omega}_{0}$ to a coordinate system that defines the vector $\boldsymbol{\Omega}_{f}$. The calculation for determining the angle $\beta_{f}$ (or $\boldsymbol{\Omega}_{f}$ ) is not straightforward, involving an iterative process. Let cartesian coordinates $\left(x^{\prime}, y^{\prime}, z^{\prime}\right)$, with the unit vectors $\left(\hat{\mathbf{x}}^{\prime}, \hat{\mathbf{y}}^{\prime}, \hat{\mathbf{z}}^{\prime}\right)$, be another cartesian coordinates with $\hat{\mathbf{z}}^{\prime}$ being parallel to $\boldsymbol{\Omega}_{f}$. For a given $\boldsymbol{\Omega}_{f}$, we can transfer the numerical solution $\mathbf{u}(x, y, z, t)$ in the coordinates $(x, y, z)$ at any instant $t$ to $\mathbf{u}^{\prime}\left(x^{\prime}, y^{\prime}, z^{\prime}, t\right)$ in the coordinates $\left(x^{\prime}, y^{\prime}, z^{\prime}\right)$ and, then, compute the quantity $Q$ defined as

$$
Q\left(\boldsymbol{\Omega}_{f}\right)=\frac{\int_{\mathcal{V}}\left|\hat{\mathbf{z}}^{\prime} \cdot \mathbf{u}^{\prime}\left(x^{\prime}, y^{\prime}, z^{\prime}, t\right)\right|^{2} \mathrm{~d} \mathcal{V}}{\int_{\mathcal{V}}|\mathbf{u}(x, y, z, t)|^{2} \mathrm{~d} \mathcal{V}}
$$

Obviously, it is the position of $\boldsymbol{\Omega}_{f}$ that determines the size of $Q$. The vector $\boldsymbol{\Omega}_{f}$ is determined, through an iterative process, such that $Q$ reaches its minimum which is typically between 0.1 and 0.2 for the nonlinear precessing solutions reported in this paper. Note that the vector $\boldsymbol{\Omega}_{f}$ obtained in this way is global and dependent only on time as illustrated in Figure 3. Our extensive calculations suggest that the size of $\beta_{f}$ strongly depends on the nonlinearity (the size of $|P o|$ ) of a precessing flow. In the limit $P o \rightarrow 0$, we always have $\boldsymbol{\Omega}_{f} \cdot \boldsymbol{\Omega}_{0} \rightarrow 0$ at any instant, i.e., $\beta_{f} \rightarrow 90^{\circ}$. This is because, 


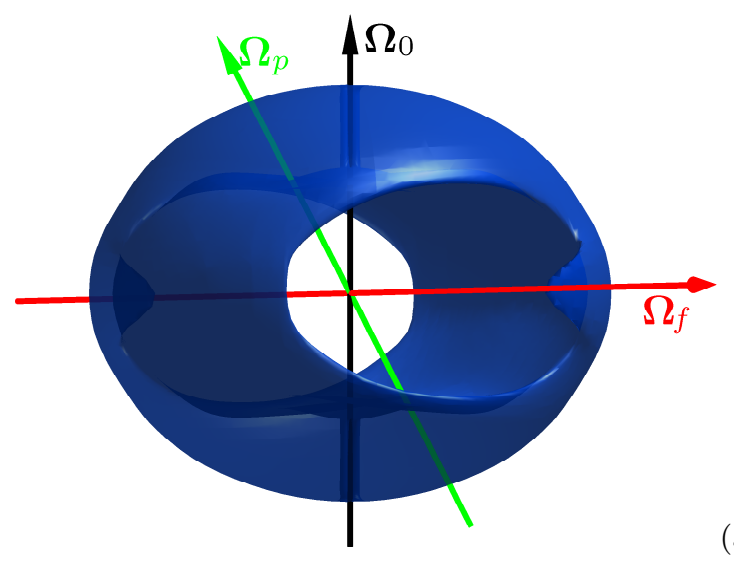

(a)
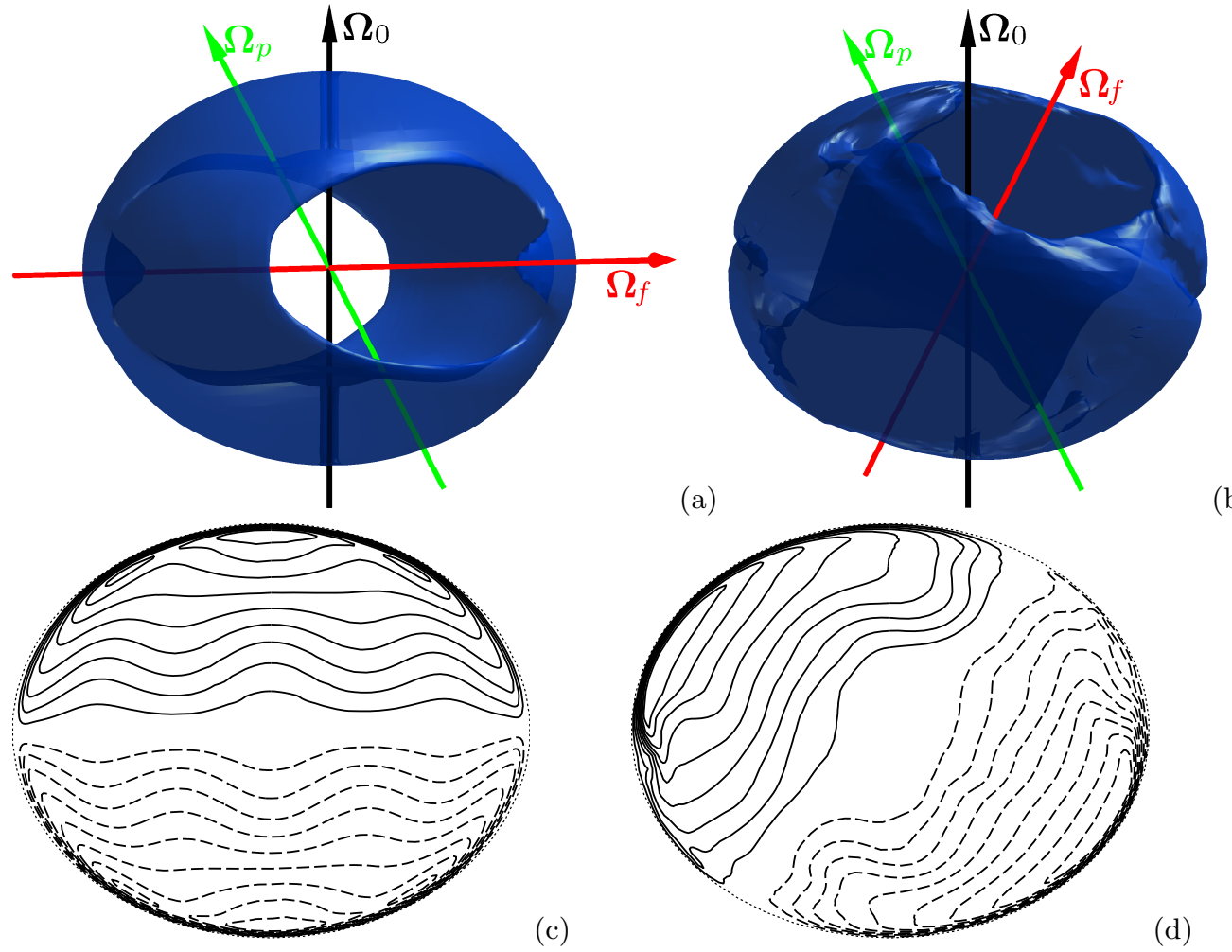

(b)

FIgURE 6 . Isosurface of the azimuthal flow together with the position of the three vectors $-\boldsymbol{\Omega}_{0}$, $\boldsymbol{\Omega}_{p}$ and $\boldsymbol{\Omega}_{f}$ - obtained with $\mathcal{E}=0.6$ and $\alpha_{p}=23.5^{\circ}$ : (a) for $P o=-0.01$ with $\beta_{f}=89^{\circ}$ and (b) for $P o=-0.2$ with $\beta_{f}=35^{\circ}$. The corresponding contours of $\hat{\phi} \cdot \mathbf{u}$ in a meridional plane: (c) for $P o=-0.01$ and (d) for $P o=-0.2$.

except for within the thin boundary layer, $\boldsymbol{\Omega}_{0} \cdot(\nabla \times \mathbf{u})=0$ where $\mathbf{u}$ is given by (3.23) and $\nabla \times \mathbf{u}$ is always perpendicular to $\boldsymbol{\Omega}_{0}$, independent of the spatial variables and, hence, global. In consequence, the mean azimuthal flow $U$ in the mantle frame, which is defined as

$$
U(s, z)=\frac{1}{2 \pi} \int_{0}^{2 \pi}\left[\frac{1}{2 \pi} \int_{0}^{2 \pi} \hat{\boldsymbol{\phi}} \cdot \mathbf{u}(s, \phi, z, t) \mathrm{d} \phi\right] \mathrm{d} t,
$$

always vanishes, i.e., $U \rightarrow 0$ when $P o \rightarrow 0$. At any fixed instant, the interior part of the expression (3.23), which is valid for $\left|P_{o}\right| \ll 1$ and $E k \ll 1$, describes fluid motion in the form of rigid-body rotation whose axis $\boldsymbol{\Omega}_{f}$ lies in the equatorial plane with $\boldsymbol{\Omega}_{f} \cdot \boldsymbol{\Omega}_{0} \rightarrow 0$.

When the nonlinear effect becomes stronger with increasing $|P o|$, the most significant change is that the direction of $\boldsymbol{\Omega}_{f}$ moves towards $\boldsymbol{\Omega}_{0}$ with the angle $\beta_{f}$ gradually decreasing from $90^{\circ}$ to a smaller value whilst the amplitude of the precessing flow may become time-dependent. Figure 4 shows kinetic energies $E_{\text {kin }}$ as a function of $t$ for several different values of $P o$ at fixed $\mathcal{E}=0.1$ (Figure $4(\mathrm{a})$ ) and $\mathcal{E}=0.6$ (Figure $4(\mathrm{~b})$ ) with $E=10^{-4}$ and $\alpha_{p}=23.5^{\circ}$. In Figure $4(\mathrm{~b})$, the special feature that $E_{\text {kin }}(P o=-0.2)>E_{\text {kin }}\left(P_{o}=-0.5\right)$ is likely to be caused by the resonance associated with $P o<0$, which was discussed by Busse (1968) and Noir et al. (2003) [see also (5.10) in Section 5]. It is interesting to notice that the variation of the strongly nonlinear flows shown in Figure 4 is much slower than the period of the main rotation. In order to reveal the spatial structure of the nonlinear precessing flow as well as the size of the angle $\beta_{f}$, we also depict (i) a three-dimensional 
isosurface of the azimuthal flow with respect to the vector $\boldsymbol{\Omega}_{f}$ and (ii) contours of the azimuthal flow in a meridional plane. Displayed in Figure 5(a) is an isosurface of the azimuthal flow, along with the three characteristic vectors $\boldsymbol{\Omega}_{0}, \boldsymbol{\Omega}_{p}$ and $\boldsymbol{\Omega}_{f}$, for the nonlinear precessionally driven flow at an instant obtained with $\mathcal{E}=0.1, E=10^{-4}$, Po $=-0.01$ and $\alpha_{p}=23.5^{\circ}$. The corresponding contours of the component $u_{\phi}$ at that instant is illustrated in Figure 5(c). For the weakly precessing flow at $|P o|=0.01$, the angle $\beta_{f}$ is estimated to be about $\beta_{f}=80^{\circ}$. When $|P o|$ increases to $0.2, \beta_{f}$ decreases to about $\beta_{f}=46^{\circ}$ which is shown in Figure $5(\mathrm{~b})$ and Figure $5(\mathrm{~d})$. It is worth mentioning that, although the kinetic energies of the precessing flow at $|P o|=0.2$ become time-dependent, the size of the angle $\beta_{f}$ remains largely unchanged at different times.

When the nonlinear effect becomes stronger with increasing $|P o|$, another change is caused by instabilities of the primary flow which are signaled by irregular variations of the kinetic energy shown in Figure 4(a). To identify the precise nature of instabilities would be a difficult task as discussed by Lorenzani and Tilgner (2001, 2003). There may exist at least three different instabilities: (i) an elliptical instability associated with the elliptical shape of bulk streamlines (Kerswell 1993); (ii) an instability originating from the interior shear caused by large deviations of the precessing flow from the uniform vorticity (Malkus 1968); and (iii) an instability due to strong shears in the thin Ekman boundary layer on the bounding surface of the container Lorenzani and Tilgner (2001). Our computational results, as suggested by Figures 5 and 6, seem to point to the third instability - which is driven by strong shears in the vicinity of the viscous boundary layer - as a possible mechanism. It should be pointed out that instabilities in the spheroidal precession problem are, by comparison, less physically significant than those taking place in the problem of thermal convection. This is because an instability in convection usually leads to a totally different flow marked by the new spatial structure while an instability in spheroidal precession only slightly modifies the spatial structure of the primary flow, which is clearly indicated in Figures 5 and 6 . A primary nonlinear effect in moderate nonlinear regimes is to reduce the size of the angle $\beta_{f}$ without dramatically changing the pattern of precessing flow. This is why our focus is placed on the size of the angle $\beta_{f}$.

The nonlinear behaviors remain largely similar for more flattened oblate spheroids. An example of the nonlinear precessing flow for $\mathcal{E}=0.6$ is shown in Figure 6 . For the weakly precessing flow at $P o=-0.01$, the angle $\beta_{f}$ is estimated to be about $89^{\circ}$ which is illustrated in Figures 6(a) and 6(c). It decreases to $\beta_{f} \approx 35^{\circ}$ (Figures 6(b) and 6(d)) when $|P o|$ increases from 0.01 to 0.2 . Although the kinetic energies $E_{\text {kin }}$ of the nonlinear precessing flow, as shown in Figure 4, may become time-dependent when $|P o|$ becomes sufficiently large, a detailed examination indicates that the main global structure of the nonlinear precessing flow is always approximately described by a time-dependent vector $\boldsymbol{\Omega}_{f}$ moving along the cone of an angle $\beta_{f}$ (depicted in Figure 3 ) whose size is primarily determined by the size of $|P o|$ at $E k \ll 1$.

It is the size of the angle $\beta_{f}$ that determines the amplitude of the mean azimuthal flow $U$ defined by equation (4.3). When $\boldsymbol{\Omega}_{f} \cdot \boldsymbol{\Omega}_{0} \approx 0$ at $0<|P o| \ll 1$, there exists no significant projection of $\boldsymbol{\Omega}_{f}$ onto the direction of $\boldsymbol{\Omega}_{0}$ and, consequently, the azimuthal mean flow $U$ is nearly zero. As the direction of $\boldsymbol{\Omega}_{f}$ for a nonlinear precessing flow shifts towards $\boldsymbol{\Omega}_{0}$ as $|P o|$ increases, a substantial projection onto the direction of $\boldsymbol{\Omega}_{0}$ results in a substantial mean flow $U$. The profiles of the mean flow $U$, which is time-independent and always westward, are shown in Figure 7 for two different cases with $\mathcal{E}=0.1$ and $\mathcal{E}=0.6$ for $\alpha_{p}=23.5^{\circ}$ and $P o=-0.2$ at $E=10^{-4}$. In both the cases, by comparing to the differential rotation discussed by Busse (1968), the mean flow $U$ in Figure 7 is westward everywhere, does not seem to exhibit singular behaviors at the critical latitudes 


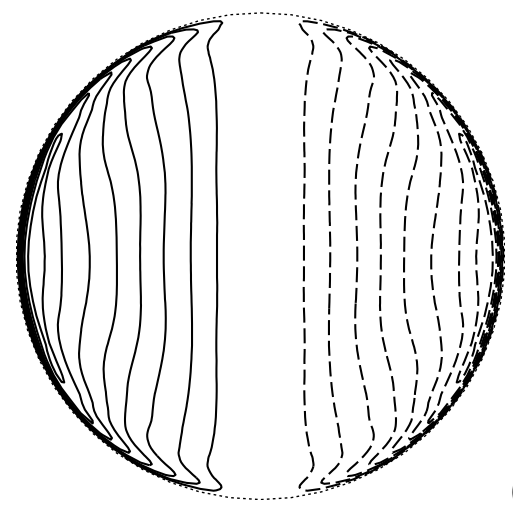

(a)

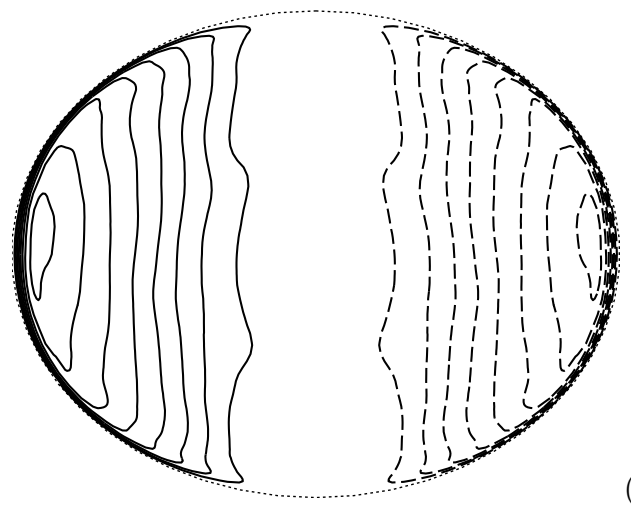

(b)

Figure 7. Contours of the mean azimuthal flow $U$ for $\alpha_{p}=23.5^{\circ}, P o=-0.2$ and $E=10^{-4}$ : (a) for $\mathcal{E}=0.1$ and (b) for $\mathcal{E}=0.6$. The mean flow $U$ represents the westward motion of fluid relative to the spheroidal container.

and has an amplitude of the same order as that of the leading-order precessing flow, i.e., $U=\mathrm{O}\left(\cos \beta_{f}|\mathbf{u}|\right)$.

The mechanism of generating a mean azimuthal flow $U$ in precessing spheroids is fundamentally different from that in spherical rotating convection. For nonlinear convection in spheres or spherical shells, it is the nonlinear interaction of spiralling convective rolls with amplitude $|\mathbf{u}|=\mathrm{O}(\epsilon)$ that, via the Reynolds stresses, generates the mean flow marked by the amplitude $U=\mathrm{O}\left(\epsilon^{2}\right)$ (Zhang 1992). More significantly, the mean azimuthal flow $U$ generated by this mechanism is distinctly marked by having both eastward and westward directions (see, for example, Liao and Zhang 2012b). For nonlinear precession in oblate spheroids, the mean azimuthal flow $U$ is produced by changing the orientation of the vector $\boldsymbol{\Omega}_{f}$ for a precessing flow with amplitude $|\mathbf{u}|=\mathrm{O}(\epsilon)$. In consequence, the mean azimuthal flow $U$ must be westward everywhere and would be of the same order as that of the precessing flow, $U=\mathrm{O}(\epsilon)$, if the angle $\beta_{f}$ is moderate. Furthermore, our computation suggests that the mean flow generated by this mechanism, driven by either prograde or retrograde precession, is distinctly characterized by having only westward direction.

\section{Summary and remarks}

We have studied, through both asymptotic analysis and direct numerical simulation, precessionally driven flows confined in an oblate spheroidal cavity of arbitrary eccentricity. Without making prior assumptions about the spatial-temporal structure of the flow, we have derived a time-dependent asymptotic solution (3.23) in the mantle frame of reference satisfying the non-slip boundary condition and valid for an oblate spheroidal cavity of arbitrary eccentricity. Fully nonlinear numerical simulation using a finite element method shows a satisfactory agreement between the asymptotic solution and the numerical simulation when $0<E k \ll 1$ and $0<|P o| \ll 1$. It is found that, when the nonlinear effect becomes strong with increasing $|P o|$, the vector $\boldsymbol{\Omega}_{f}$ moves towards $\boldsymbol{\Omega}_{0}$ with the size of the angle $\beta_{f}$ gradually decreasing from nearly $90^{\circ}$ to as small as about $35^{\circ}$. The substantial change of the orientation of $\boldsymbol{\Omega}_{f}$ results in a large-amplitude, time-independent azimuthal mean flow in the form of westward fluid motion. While the nonlinear effect in thermal convection in spheres can generate a mean azimuthal flow marked by both eastward and westward directions, the azimuthal mean flow $U$ produced by precession in oblate spheroids is always westward, which is clearly shown in Figure 7. 


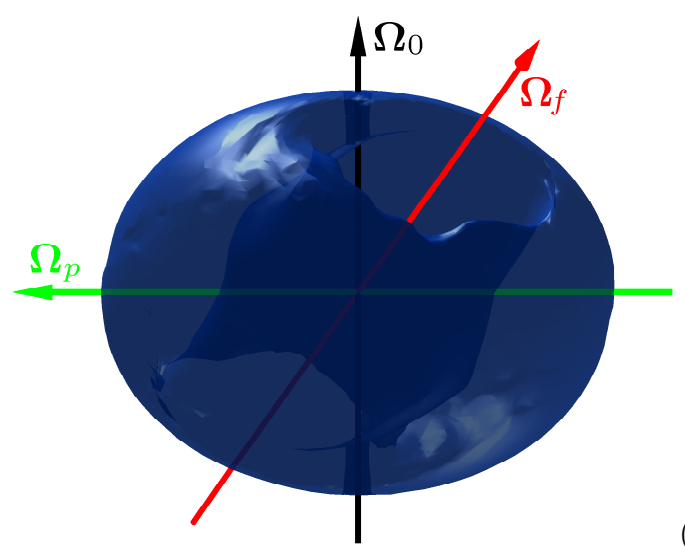

(a)

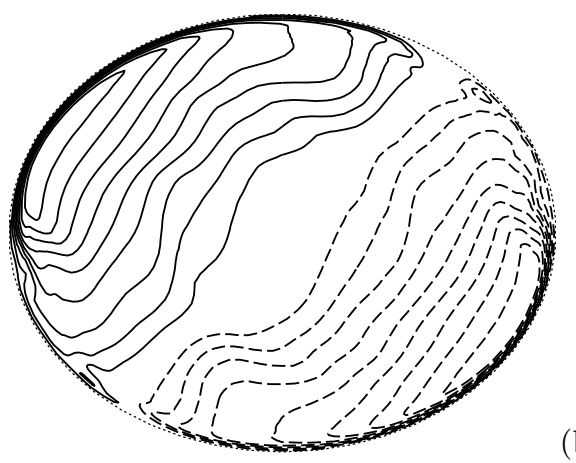

(b)

FIgURE 8. (a) Isosurface of the azimuthal flow together with the position of the three vectors, $\boldsymbol{\Omega}_{0}, \boldsymbol{\Omega}_{p}$ and $\boldsymbol{\Omega}_{f}$, obtained for $\mathcal{E}=0.6, E k=10^{-4}, P o=-0.1$ and $\alpha_{p}=90^{\circ}$ with $\beta_{f} \approx 45^{\circ}$, and (b) the corresponding contours of $\hat{\boldsymbol{\phi}} \cdot \mathbf{u}$ in a meridional plane.

We have concentrated on the cases with $\alpha_{p}=23.5^{\circ}$ and $P o<0$ in our discussion. An extensive simulation with different sizes of $\alpha_{p}$ was also performed using both positive and negative values of $P_{o}$ at $E k \ll 1$, showing a largely similar feature to that depicted in Figures 5 and Figure 6 . For example, the positions of the three vectors, $\boldsymbol{\Omega}_{0}, \boldsymbol{\Omega}_{p}$ and $\boldsymbol{\Omega}_{f}$, along with the contours of $\hat{\boldsymbol{\phi}} \cdot \mathbf{u}$ in a meridional plane, are shown in Figure 8 for $\alpha_{p}=90^{\circ}$ with $\mathcal{E}=0.6$ and $P o=-0.1$ in which case the angle $\beta_{f}$ is approximately about $45^{\circ}$. Displayed in Figure 9 is a summary of the angle $\beta_{f}$ as a function of $P o$ for two different eccentricities, $\mathcal{E}=0.1$ and $\mathcal{E}=0.6$, at $E k=10^{-4}$. For $\mathcal{E}=0.1$, the minimum size of $\beta_{f}$ is about $46^{\circ}$ when $P o \approx-0.23$ while the minimum $\beta_{f}$ for $\mathcal{E}=0.6$ reaches about $25^{\circ}$ when $P_{o} \approx-0.17$.

An important unanswered question is concerned with (i) the precise mechanism that determines the size of the angle $\beta_{f}$ and (ii) the minimum $\beta_{f}$ that can be attained in precessing spheroids at $E k \ll 1$. Evidently, the nonlinear effect within the spheroidal viscous boundary layer is likely to play a key role. Understanding the precise mechanism and, then, determining the minimum $\beta_{f}$ for given $\mathcal{E}$ at $E k \ll 1$ would require the asymptotic analysis of fully nonlinear spheroidal boundary layers, which represents a difficult mathematical problem.

It is the extremely small size of the Ekman number, a consequence of the Earth's rapid rotation and the small viscosity in its liquid core, that causes severe difficulties in numerical modelling of the Earth's core dynamics. Even using modern powerful parallel supercomputers, we still cannot achieve the geophysically realistic value which is $\mathrm{O}\left(10^{-9}\right) \leqslant E k \leqslant \mathrm{O}\left(10^{-15}\right)$. In this sense, the analytical expression (3.23) valid for an arbitrarily small Ekman number would be particularly useful. For example, a geophysically important question is whether the precessionally driven flow in the Earth's fluid core can produce westward fluid motion with amplitude $\mathrm{O}\left(10^{-4} \mathrm{~m} / \mathrm{s}\right)$. If we assume that (i) the expression (3.23), as demonstrated in Figure 2, gives a correct amplitude of the weakly nonlinear precessing flow for $0<E k \ll 1$ and (ii) the primary nonlinear effect at $0<E k \ll 1$ is to reduce the size of the angle $\beta_{f}$, we would be able to provide an estimate for the amplitude of westward motion in the Earth's fluid core driven by its precession. Define the dimensional speed of the westward fluid motion, $V$, of the precessing flow in 


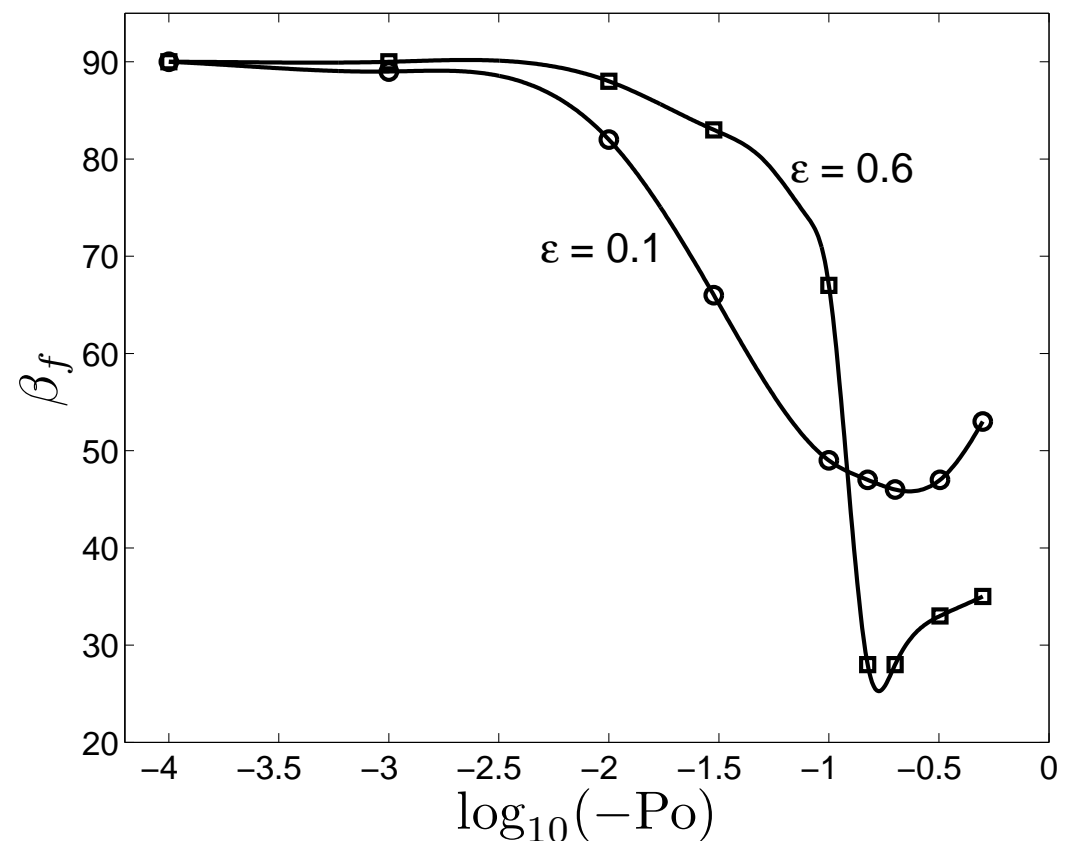

Figure 9. A summary of the angle $\beta_{f}$ is shown as a function of $P o$ for two different eccentricities at $E k=10^{-4}$ : the circles represent the solutions for $\mathcal{E}=0.1$ while the squares denote the solutions for $\mathcal{E}=0.6$.

the Earth's spheroidal fluid core as

$$
V=\cos \beta_{f}\left[\frac{1}{\mathcal{V}} \int_{\mathcal{V}}|\mathbf{u}|^{2} \mathrm{~d} \mathcal{V}\right]^{1 / 2}\left(r_{o} \Omega_{0}\right),
$$

where $\mathbf{u}$ is given by $(3.23), \mathcal{V}=4 \pi\left(1-\mathcal{E}^{2}\right) / 3, r_{o}=3.485 \times 10^{6} \mathrm{~m}, \Omega_{0}=7.27 \times 10^{-5} / \mathrm{s}$. Taking $|P o|=10^{-7}, \alpha_{p}=23.5^{\circ}, \mathcal{E}=0.082$ and $E k=10^{-9}$ appropriate for the Earth together with its typical speed $V \approx 10^{-4} \mathrm{~m} / \mathrm{s}$, we obtain $\beta_{f}=60^{0}$ which is not unreasonable. In other words, the Earth's precession, via both the viscous and topographical coupling between its fluid core and solid mantle, is capable of driving a sufficiently strong westward flow required to explain the geomagnetic secular variation if we assume that the angle $\beta_{f}$ can be reduced, by nonlinear effects, from $90^{\circ}$ to about $60^{\circ}$.

Though the mathematical analysis of the precessing flow in the mantle frame is usually complicated and lengthy for spheroidal geometry, it offers a desirable potential that we may unify the mathematical theories of buoyancy-driven convection and precessionally driven flow in the same frame. This unified approach, considering that there exists a severe restriction on the thermal power available for sustaining the geodynamo, is particularly significant for constructing a planetary dynamo model in that both driving mechanisms, convection and precession, are energetically important.

It is desirable to discuss the possibility of transforming the weak precession solutions derived by Busse (1968) valid for $\mathcal{E} \ll 1$ and $\alpha_{p} \ll 1$ in the precession frame to (3.25) in the mantle frame obtained from the general solution (3.23) via the expansion of small $\mathcal{E}$. Such a transformation, if possible, would allow a precise comparison between the mantleframe solution (3.25) and the precession-frame solution. For this purpose, it is desirable to provide a brief summary of the Busse (1968)'s solution largely using his notation. By assuming that the precessing flow is stationary, the governing equations for the velocity 
vector $\mathbf{q}$ and the pressure $p_{q}$ in the precession frame are

$$
\begin{gathered}
\mathbf{q} \cdot \nabla \mathbf{q}+2 \Omega \times \mathbf{q}=-\nabla p_{q}+E k \nabla^{2} \mathbf{q}, \\
\nabla \cdot \mathbf{q}=0,
\end{gathered}
$$

which can be compared to (2.1)-(2.2) in the mantle frame. Busse (1968) solved (5.1)-(5.2) for $0<E k \ll 1$ and $0<|\boldsymbol{\Omega}| \ll 1$ subject to the non-slip boundary condition

$$
\mathbf{q}=\mathbf{k} \times \mathbf{r}
$$

on the bounding surface $\mathcal{S}$ of the container, where $\mathbf{k}$ being the unit vector in the direction of the basic rotation. The boundary condition (5.3) in the precession frame can be compared to (2.4) in the mantle frame. Moreover, the precession vector $\Omega$ in $(5.1)$ is independent of time with $|\boldsymbol{\Omega}|=|P o|$. The mathematical analysis in the precession frame becomes particularly convenient by further postulating that the precessing flow is not only stationary but also in the form of rigid-body rotation given by

$$
\mathbf{q}=\omega \times \mathbf{r},
$$

where $\boldsymbol{\omega}$ is a constant vector to be determined. When $0<|P o| \ll 1$, the weakly precessing flow $\mathbf{q}$ is written in the form

$$
\mathbf{q}=\mathbf{q}_{i}+\tilde{\mathbf{q}}=\boldsymbol{\omega} \times \mathbf{r}+\epsilon \tilde{\mathbf{q}}_{0}^{(1)}+\ldots
$$

where $\mathbf{q}_{i}($ or $\boldsymbol{\omega} \times \mathbf{r})$ denotes the interior flow, the boundary layer flow $\tilde{\mathbf{q}}\left(\right.$ or $\left.\tilde{\mathbf{q}}_{0}^{(1)}\right)$ satisfies the condition

$$
\epsilon \tilde{\mathbf{q}}_{0}^{(1)}=(\mathbf{k}-\boldsymbol{\omega}) \times \mathbf{r}
$$

on the bounding surface $\mathcal{S}$ and $\epsilon$ is small and assumed to be $\epsilon^{2}=|\mathbf{k}-\boldsymbol{\omega}|^{2}$. Expansion (5.5) in the precession frame can be compared with our most general expansions (3.1) and (3.2) that make no prior assumptions about the spatial structure of the flow in the mantel frame. Busse (1968) showed that $\tilde{\mathbf{q}}_{0}^{(1)}$ in (5.5) is given by

$$
\begin{aligned}
\hat{\mathbf{n}} \times \tilde{\mathbf{q}}_{0}^{(1)}+\mathrm{i} \tilde{\mathbf{q}}_{0}^{(1)} & =\left[\hat{\mathbf{n}} \times\left(\boldsymbol{\omega}_{+} \times \mathbf{r}\right)+\mathrm{i}\left(\boldsymbol{\omega}_{+} \times \mathbf{r}\right)\right] \mathrm{e}^{-\kappa_{+} \xi} \\
& +\left[\hat{\mathbf{n}} \times\left(\boldsymbol{\omega}_{-} \times \mathbf{r}\right)+\mathrm{i}\left(\boldsymbol{\omega}_{-} \times \mathbf{r}\right)\right] \mathrm{e}^{-\kappa_{-} \xi} \\
& -\frac{1}{\epsilon}\left(1-\frac{\mathbf{k} \cdot \boldsymbol{\omega}}{\omega^{2}}\right)[\hat{\mathbf{n}} \times(\boldsymbol{\omega} \times \mathbf{r})+\mathrm{i}(\boldsymbol{\omega} \times \mathbf{r})] \mathrm{e}^{-\kappa \xi}
\end{aligned}
$$

where

$$
\boldsymbol{\omega}_{ \pm}=-\frac{\boldsymbol{\omega} \times(\boldsymbol{\omega} \times \mathbf{k})}{2 \omega^{2} \epsilon} \pm\left(\frac{\boldsymbol{\omega} \times \mathbf{k}}{2 \omega \epsilon}\right) \mathrm{i}
$$

and $\kappa_{+}, \kappa_{-}$and $\kappa$ are determined by the relations

$$
\begin{aligned}
\kappa_{ \pm}^{2}-2 \mathrm{i}(\boldsymbol{\Omega}+\boldsymbol{\omega}) \cdot \hat{\mathbf{n}} & = \pm \mathrm{i} \omega, \\
\kappa^{2}-2 \mathrm{i}(\boldsymbol{\Omega}+\boldsymbol{\omega}) \cdot \hat{\mathbf{n}} & =0,
\end{aligned}
$$

taking the root with positive real part, while the angular velocity $\boldsymbol{\omega}$ of the fluid in (5.5) is

$$
\begin{aligned}
\boldsymbol{\omega} & =\mathbf{k} \omega^{2}+\omega^{2} \\
& \times \frac{\mathbf{k} \times \boldsymbol{\Omega} 2.62(E k \omega)^{1 / 2}+\mathbf{k} \times(\boldsymbol{\Omega} \times \mathbf{k})\left[0.259(E k / \omega)^{1 / 2}+\eta \omega^{2}+\mathbf{k} \cdot \boldsymbol{\Omega}\right]}{\left[0.259(E k / \omega)^{1 / 2}+\eta \omega^{2}+\mathbf{k} \cdot \boldsymbol{\Omega}\right]^{2}+(2.62)^{2} E k \omega}
\end{aligned}
$$

where the ellipticity $\eta$ and the eccentricity $\mathcal{E}$ are related by $\mathcal{E}^{2}=\eta(2-\eta)$ with $\eta \ll 1$, 
and $\omega^{2}=1-\epsilon^{2}$. In the weak precession limit, the term $\mathbf{k} \cdot \boldsymbol{\Omega}$, along with the associated resonance when $\mathbf{k} \cdot \boldsymbol{\Omega}<0$, can be removed from (5.7).

Can $\mathbf{q}$ expressed implicitly by (5.5) in the precession frame - for which $\tilde{\mathbf{q}}_{0}^{(1)}$ is given by (5.6) and $\boldsymbol{\omega}$ by (5.7) - be easily transformed to $\mathbf{u}$ expressed explicitly by (3.25) in the mantle frame? It is found that such a transformation is, at least, not straightforward. In fact, there are no existing studies in literature that demonstrate this transformation can be readily performed in any geometries. The major complication stems from the facts that (i) the two precession solutions, $\mathbf{q}$ given by (5.5) and $\mathbf{u}$ given by (3.25), for $0<E k \ll 1$ are derived from the asymptotic match between the two different complicated boundary solutions and the relatively simple interior solutions, and (ii) the two problems - the boundary layer and the interior - are intricately coupled and inseparable. We seem to reach a conclusion that there exists no simple transformation between (5.5) in the precession frame and (3.25) in the mantle frame.

Finally, we would like to point out that the term $\mathbf{k} \cdot \boldsymbol{\Omega}$ in (5.7) of the Busse (1968)'s solution valid for $\alpha_{p} \ll 1$ can be readily included in our general asymptotic solution in the mantle frame of reference. When both the precession rate $P o$ and the precession angle $\alpha_{p}$ are sufficiently small [see (3.20) in Busse (1968)] with $\left|P o \widehat{\boldsymbol{\Omega}}_{p} \times \mathbf{u}\right| \gg|\mathbf{u} \cdot \nabla \mathbf{u}|$, the momentum equation (2.5) becomes

$$
\frac{\partial \mathbf{u}}{\partial t}+2 \hat{\mathbf{z}} \times \mathbf{u}=-\nabla p+E k \nabla^{2} \mathbf{u}-\hat{\mathbf{z}}\left(2 s P o \sin \alpha_{p} \mathrm{e}^{\mathrm{i} \phi}\right) \mathrm{e}^{\mathrm{i} t}-2 P o \widehat{\boldsymbol{\Omega}}_{p} \times \mathbf{u} .
$$

It follows that the solvability condition (3.13) requires an extra term representing perturbations to the Coriolis force:

$$
\begin{aligned}
& -\left(\frac{\mathrm{i} \mathcal{E}^{2}}{2-\mathcal{E}^{2}}\right) \mathcal{A}_{110} \int_{\mathcal{V}}\left|\mathbf{u}_{110}\right|^{2} \mathrm{~d} \mathcal{V}+\int_{\mathcal{S}}\left\{\left[p_{110}^{*}\right]_{\mathcal{S}} \hat{\mathbf{n}} \cdot \widehat{\mathbf{u}}\right\} \mathrm{d} \mathcal{S} \\
& \quad=\left(\frac{4 \pi \mathrm{i} P o \sin \alpha_{p}}{5}\right)\left(2-\mathcal{E}^{2}\right) \sqrt{1-\mathcal{E}^{2}}+\left(\frac{\mathrm{i} 2 P o \cos \alpha_{p}}{2-\mathcal{E}^{2}}\right) \mathcal{A}_{110} \int_{\mathcal{V}}\left|\mathbf{u}_{110}\right|^{2} \mathrm{~d} \mathcal{V},
\end{aligned}
$$

which introduces an extra term in the expression for $\mathcal{A}_{110}$ given by (3.22) which now becomes

$$
\mathcal{A}_{110}=\frac{16 \sqrt{2} P_{o} \sin \alpha_{p}\left(1-\mathcal{E}^{2}\right)}{45 \sqrt{E k}\left(\mathcal{I}_{r}+\mathrm{i} \mathcal{I}_{i}\right) \sqrt{1-\mathcal{E}^{2}}-6 \sqrt{2}\left(2-\mathcal{E}^{2}\right)\left(\mathcal{E}^{2}+2 P o \cos \alpha_{p}\right)} .
$$

This suggests that the resonance may occur when $P o<0$ such that $\left(\mathcal{E}^{2}+2 P o \cos \alpha_{p}\right)=0$.

KZ is supported by UK STFC and NERC grants. KHC is supported by Hong Kong RGC grant/700310 and XL is supported by NSFC/11133004 and Chinese Academy of Sciences under grant number KZZD-EW-01-3. The numerical computation is supported by Shanghai Supercomputer Center.

\section{REFERENCES}

Boisson, J., CÉbron, D.C., Moisy, F. And Cortet, P.-P. 2012. Earth rotation prevents exact solid-body rotation of fluids in the laboratory. Europhysics Letters 98, 59002.

Bullard E.C. 1949. The magnetic flux within the earth. Proceedings of the Royal Society A 197, 433-453.

Busse, F.H. 1968. Steady fluid flow in a precessing spheroidal shell. J. Fluid Mech. 136, 739-751.

Cébron, D.C., Le Bars, M. And Meunier, P. 2010. Tilt-over mode in a precessing triaxial ellipsoid. Physics of Fluids 22, 116601.

Chan, K., Zhang K., And Liao, X. 2010. An EBE finite element method for simulating non- 
linear flows in rotating spheroidal cavities. International Journal for Numerical Methods in Fluids 63, 395-414.

Cui, Z., Zhang, K. And Liao, X. 2013. On the completeness of inertial wave modes in rotating annular channels. Geophysical and Astrophysical Fluid Dynamics doi/full/10.1080/03091929.2013.821117.

Dwyer, C.A., Stevenson, D.J. And Nimmo, F. 2011. A long-lived lunar dynamo driven by continuous mechanical stirring. Nature, 479, 212-214.

Goto, S., Ishit, N., KidA, S. And NishiokA, M. 2007. Turbulence generator using a precessing sphere. Phys. Fluids 19, 061705.

Greenspan, H. P. 1968. The Theory of Rotating Fluids. Cambridge University Press.

Hollerbach, R. AND Kerswell R. R. 1995. Oscillatory internal shear layers in rotating and precessing flows. J. Fluid Mech. 298, 327-339.

Hollerbach, R., Nore, C., Marti, P., Vantieghem, S., Luddens, F. and Léorat, J. 2013. Parity-breaking flows in precessing spherical containers. Physical Review E 87, 053020.

Kerswell, R.R. 1993. The instability of precessing flow. Geophysical and Astrophysical Fluid Dynamics 72, 107-144.

KeRswell, R.R. 1996. Upper bounds on the energy dissipation in turbulent precession. J. Fluid Mech. 321, 335-370.

KIDA, S. 2011. Steady flow in a rapidly rotating sphere with weak precession. J. Fluid Mech. 680, 150-193.

Kong, D., Zhang, K. And Schubert, G. 2010. Shapes of two-layer models of rotating planets. J. Geophy. Research 115, E12003

Kong, D., Zhang, K., Schubert, G., Anderson, J. 2013. A three-dimensional numerical solution for the shape of a rotationally distorted polytrope of index unity. Astrophys. J. 763, 116, doi:10.1088/0004-637X/763/2/116.

LiAO, X. AND ZHANG, K. 2012a. On flow in weakly precessing cylinders: the general asymptotic solution. J. Fluid Mech. 709, 610-621

LiAO, X. AND Zhang, K. 2012b. Asymptotic solutions of differential rotation driven by convection in rapidly rotating fluid spheres with the non-slip boundary condition. Geophys. Astrophys. Fluid Dyn. 692, 420-445

Lorenzani, S. And Tilgner, A. 2001. Fluid instabilities in precessing spheroidal cavities. J. Fluid Mech. 447,111-128.

Lorenzani, S. And Tilgner, A. 2003. Inertial instabilities of fluid flow in precessing spheroidal shells. J. Fluid Mech. 492, 363-379.

Malkus W.V.R. 1968. Precession of the earth as the cause of geomagnetism. Science 136, 259-264.

Noir, J., JAult, D. And Cardin, P. 2001. Numerical study of the motions within a slowly precessing sphere at low Ekman number. J. Fluid Mech. 437, 283-299.

Noir, J., Cardin, P., JAult, D. And Masson J.P. 2003. Experimental evidence of non-linear resonance effects between retrograde precession and the tilt-over mode within a spheroid. Geophysical Journal International 154, 407-416.

Poincaré, H., 1910. Sur la précession des corps déformables. Bull. Astron., 27, 321-356.

Pozzo, M., Davies, C., Gubbins, D. And Alfe, D. 2012. Thermal and electrical conductivity of iron at Earths core conditions Nature 458, 355-358.

Roberts, P.H. And Stewartson, K. 1965. On the motion of a liquid in a spheroidal cavity of a precessing rigid body: II. Mathematical Proceedings of the Cambridge Philosophical Society 61, 279-288.

Stewartson K. And Roberts P.H. 1963. On the motion of a liquid in a spheroidal cavity of a precessing rigid body. J. of Fluid Mech. 17, 1-20.

Tilgner, A. And Busse, F.H. 2001. Fluid flows in precessing spherical shells. J. Fluid Mech. 426, 387-396.

Tilgner, A. 2005. Precession driven dynamos. Physics of Fluids 17, 034104-034106.

Tilgner, A. 2007. Rotational Dynamics of the core. in Core Dynamics, ed. by G. Schubert. Treatise of Geophysics, vol. 8 (Elsevier, Amsterdam, 2007), pp. 207-243.

Triana, S. A., Zimmerman, D. S. And Lathrop D. P. 2012. Precessional states in a laboratory model of the Earth's core. J. Geophys. Res. 117, B04103. 
VANYO, J.P. AND Likins P.W. 1972. Rigid-body approximation to turbulent motion in a liquidfilled, precessing, spherical cavity. J. Appl. Mech. 39, 19-24.

Vanyo, J.P., Wilde, P., Cardin, P. And Olson, P. 1995. Experiments on precessing flows in the Earth's liquid core. Geophysical Journal International 121, 136-142.

Wei, X. And Tilgner, A. 2013. Stratified precessional flow in spherical geometry. J. Fluid Mech. 718, DOI: http://dx.doi.org/10.1017/jfm.2013.68

Wu, C.C. And Roberts, P.H. 2009. On a dynamo driven by topographic precession. Geophysical and Astrophysical Fluid Dynamics 103, 467-501.

ZHANG, K. 1992. Spiralling columnar convection in rapidly rotating spherical fluid shells. $J$. Fluid Mech. 236, 535-556.

Zhang, K., Liao, X. And Earnshaw, P. 2004. On inertial waves and oscillations in a rapidly rotating fluid spheroid. J. Fluid Mech. 504, 1-40.

Zhang, K., Chan, K. And LiaO X. 2010 On fluid flows in precessing spheres in the mantle frame of reference. Physics of Fluids. 22, 116604 